\title{
Gyrokinetic study of the role of $\beta$ on electron particle transport in tokamaks
}

\author{
T. Hein ${ }^{1}$, C. Angioni ${ }^{1}$, E. Fable ${ }^{1}$ and J. Candy ${ }^{2}$ \\ ${ }^{1}$ Max-Planck-Institut für Plasmaphysik, IPP-EURATOM Association, D-85748 Garching bei \\ München, Germany \\ ${ }^{2}$ General Atomics, P.O. Box 85608, San Diego, United States of America
}

(December 27, 2010)

Electromagnetic effects on the radial transport of electrons in the core of tokamak plasmas are studied by means of linear and nonlinear gyrokinetic simulations with the code GYRO (J. Candy and R. E. Waltz, J. Comput. Phys. 186, $545(2003))$ and by an analytical derivation. The impact of a finite $\beta$, that is a finite ratio of the plasma pressure to the magnetic pressure, is considered on the fluctuations of the magnetic field through Ampére's law, as well as on the geometrical modification of the vertical drift produced by the Shafranov shift in the magnetic equilibrium, which, for realistic descriptions, has to be included in both electrostatic and electromagnetic modeling. The condition of turbulent particle flux at the null, which allows the determination of stationary logarithmic density gradients when neoclassical transport and particle sources are negligible, is investigated for increasing values of $\beta$, in regimes of ion temperature gradient and trapped electron mode turbulence. The loss of adiabaticity of passing electrons produced by fluctuations in the magnetic vector potential produces an outward convection. When the magnetic equilibrium geometry is kept fixed, this induces a strong reduction of the stationary logarithmic density gradient with increasing $\beta$. This effect is partly compensated by the geometrical effect on the vertical drift. This compensation effect however is significantly weaker in nonlinear simulations as compared to quasi-linear calculations. A detailed comparison between quasi- 
linear and nonlinear results reveals that the predicted value of logarithmic density gradient is highly sensitive on the assumptions on the wave number spectrum applied in the quasi-linear model. The qualitative consistency of the theoretical predictions with the experimental results obtained so far on the dependence of density peaking on $\beta$ is discussed by considering the additional impact, with increasing $\beta$, of a particle source delivered by neutral beam injection heating. (Some figures in this article are in color only in the electronic version)

\section{INTRODUCTION}

The increasing interest in high $\beta_{\mathrm{N}}$ scenarios, like the hybrid scenario with $\beta_{\mathrm{N}}$ up to 3 [1-3], for the operation of future fusion experiments like ITER [4] motivates the study of the impact of $\beta$ on the electron particle transport in tokamak plasmas. Here, the normalized ratio of plasma kinetic pressure to magnetic pressure is defined as $\beta_{\mathrm{N}}=\beta /\left(I_{\mathrm{P}} / a B_{\mathrm{T}}\right)$ with $\beta=\langle p\rangle /\left(B_{\mathrm{T}}^{2} / 8 \pi\right)$ where $\langle p\rangle$ is the volume average kinetic pressure, $I_{\mathrm{p}}$ the plasma current, $a$ the plasma minor radius and $B_{\mathrm{T}}$ the toroidal magnetic field. Particle transport determines the peaking of the density profile, which directly impacts the fusion power, since this is proportional to the density squared. The interplay between these parameters is underlined by recent studies pointing out that the cost of fusion electricity will be proportional to $\beta_{\mathrm{N}}^{-0.4}\left(n / n_{\mathrm{G}}\right)^{-0.3}[5]$, where $n_{\mathrm{G}}$ is the Greenwald density limit [6]. Thus, operational scenarios combining both high $\beta_{\mathrm{N}}$ and high densities are required to reach the goal of an economically viable energy source.

A rather large amount of studies performed during the last decade on both the experimental and the theoretical standpoints strongly supports the idea that particle transport in the core of tokamaks is produced by microturbulence, mainly due to ion temperature gradient (ITG) and trapped electron modes (TEM), see [7] and references therein. However, 
only a rather limited amount of experimental studies have been performed so far specifically on the dependence of electron particle transport and density peaking on $\beta$. This is perhaps also a consequence of the fact that from the experimental side, statistical analysis over large data sets have found weak effects of $\beta$ on the density peaking in general [8-10]. Interestingly, however, an increase of $\beta$ in all these studies has been found to be correlated with a reduction, albeit weak, of the density peaking. From the theoretical standpoint, electromagnetic effects on particle transport have been investigated with both fluid [11] and gyrokinetic [12] approaches. In [11], a quasi-linear study has revealed the existence of a convective contribution due to electromagnetic induction which is directed outward in the case of ITG modes, and which can reverse direction and become a pinch in the case of TEM. This result is consistent with the nonlinear gyrokinetic results presented in [12], where in an ITG turbulence simulation the increase of $\beta_{\mathrm{e}}$ is found to strongly affect the particle flux, and to reverse its direction from inward, in the electrostatic case, to outward when $\beta_{\mathrm{e}}$ exceeds the experimentally relevant value of $0.5 \%$. In both these previous works, the magnetic equilibrium parameters were not varied consistently with increasing $\beta_{\mathrm{e}}$. Those theoretical results indicate a rather strong impact of electromagnetic effects on particle transport, which might be interpreted as being in disagreement with the experimental observations obtained so far, although qualitatively consistent with the theoretical prediction of a reduction of density peaking with increasing $\beta$.

In the present study, we investigate specifically the effect of $\beta$ on particle transport considering both the electromagnetic effect due to the inclusion in the turbulent fields of the magnetic field fluctuations given by the solution of the Ampére's law, and the geometrical (electrostatic) effect produced by an increase of $\beta$ in the magnetic equilibrium, which affects the vertical drift. The investigation of both these two effects allows us to make simulations in which the problem of the $\beta$ dependence of density peaking in experimental conditions can be addressed in a realistic way. In fact, these two effects are unavoidably combined in an experiment, while they can be separated in theoretical studies, which are appropriate and interesting for specific analysis. In the following Sections, results from linear and nonlinear 
gyrokinetic simulations performed with the code GYRO [13,14] are presented, and the underlying physics is explained by means of an analytical derivation in which both the $E \times B$ and the magnetic flutter transport are computed starting from a formal analytical solution of the gyrokinetic equation. Magnetic flutter is caused by the fluctuating magnetic field in the direction perpendicular to that of the equilibrium field [15-17]. A quasi-linear approach is applied, justified by present knowledge of turbulent transport in the core of tokamak plasmas, which reveals that most of the main features obtained in nonlinear simulations are well captured by quasi-linear models [18-20]. In addition, comparisons among different assumptions for the wave number spectrum in quasi-linear calculations and with nonlinear results, which require very high computational effort particularly for electromagnetic cases so far, show the critical role played by this ingredient in the quasi-linear models for the prediction of the logarithmic density gradients at the null of the flux. This quantity turns out to be a particularly well suited figure of merit for models of the wave number spectrum assumed in quasi-linear transport calculations.

The problem of the effects of $\beta$ on electron particle transport is studied in the present paper as follows. In the next Section, an analytical calculation is presented by which expressions of the electromagnetic effects on particle fluxes are derived and directly compared with the numerical results, which allows the identification of the main physical mechanisms at play. In Section III, a set of quasi-linear calculations with GYRO show the impact of $\beta_{\mathrm{e}}$ on the logarithmic density gradient at the null of the particle flux, considering separately both the effect due to magnetic field fluctuations and the effect due to the geometrical modification of the curvature drift produced by the compression of the magnetic flux surfaces with increasing $\beta$. Both collisionless and collisional simulations are performed, given the important role played by collisionality on particle transport [21]. In Section IV, a realistic case with typical parameters of a high confinement $(\mathrm{H}-)$ mode at mid-radius is considered and the effect of $\beta$ on the logarithmic density gradient at the null of the particle flux is computed with both linear and nonlinear gyrokinetic calculations including a detailed comparison between quasi-linear and nonlinear spectra and addressing the problem of the comparison of 
the theoretical results with the experiment. Section V draws the main conclusions of this work.

\section{ANALYTICAL DESCRIPTION OF ELECTROMAGNETIC EFFECTS ON PARTICLE TRANSPORT}

A detailed analytical description of particle transport is presented, in which electromagnetic effects provided by Ampère's law with a finite value of $\beta_{\mathrm{e}}$ are included. In the main part of this Section it will be shown that the most significant effect is on passing particles, consistently with a previous fluid calculation [11]. The passing particle flux is finite due to the loss of adiabaticity induced by the additional $\left(v_{\|} / c\right) A_{\|}$term in the gyrokinetic equation of motion. The loss of adiabaticity of passing electrons was also pointed out in [22], and has a strong influence in ITG dominated microturbulence. A set of subsections has been included with the aim of discussing the separation of electromagnetic fluxes into their $\mathrm{E} \times \mathrm{B}$ and flutter components, the impact of shear and the pressure gradient parameter $\alpha$, the role of collisions and the behavior in TEM dominated microturbulence. The parameter $\alpha=-\left(8 \pi q^{2} R / B_{0}^{2}\right) \partial_{r} p$, where $B_{0}$ is the magnetic field strength ( $\theta$ and $r$ independent $)$ at the reference major radius $R$, encapsulates the pressure gradient contribution to the curvature drift in the $s-\alpha$ geometry model of circular flux surfaces in the large aspect ratio limit [23]. Here, $R$ is defined as the effective major radius at the center of the flux surface, and $\partial_{r}$ denotes the derivative in the radial direction with respect to the minor radius $r$, which is the half width of the flux-surface at the elevation of the centroid, as defined in [24].

Following the works of [25-28], the linear gyrokinetic equation for a particle species $\sigma$ is given by an evolution equation for the non-adiabatic component of the perturbed distribution

function $\tilde{f}_{\sigma}$, i.e. $\tilde{h}_{\sigma}=\tilde{f}_{\sigma}+F_{0, \sigma} Z_{\sigma} e \tilde{\phi} / T_{\sigma}$, where $\tilde{\phi}$ is the fluctuating electrostatic potential. $F_{0, \sigma}$ is the unperturbed Maxwellian distribution function. $Z_{\sigma}$ and $T_{\sigma}$ are the charge and the temperature of the considered species, respectively. By spectral expansion in Fourier space, the gyrokinetic equation for the perturbed distribution function $\tilde{h}_{k}$ at the wave number $k_{y}$, 
binormal to the unperturbed magnetic field, reads

$$
\begin{aligned}
& {\left[\omega_{r, k}+i\left(\gamma_{k}+\nu_{k}\right)-k_{\|} v_{\|}-\omega_{d, k} / Z_{\sigma}\right] \tilde{h}_{k, \sigma}=} \\
& \left\{\omega_{r, k}+i \gamma_{k}-\frac{\omega_{D, k}}{Z_{\sigma}}\left[\frac{R}{L_{n, \sigma}}+\left(\frac{E}{T_{\sigma}}-\frac{3}{2}\right) \frac{R}{L_{T, \sigma}}\right]\right\} \frac{Z_{\sigma} e}{T_{\sigma}} F_{0} J_{0, \sigma} \tilde{U}_{k}
\end{aligned}
$$

where $\omega_{r}$ and $\gamma$ are respectively the real part and the growth rate in the complex eigenfrequency $\omega$. The subscript $\|$ denotes the direction parallel to the unperturbed background magnetic field, and $E$ is the kinetic energy of a particle. The zero-order Bessel function $J_{0, \sigma} \equiv J_{0}\left(k_{\perp} v_{\perp} / \Omega_{c, \sigma}\right)$, where $\Omega_{c, \sigma}$ is the gyration frequency, produces the gyro-average in Fourier space, while $\omega_{D, k}=k_{\mathrm{y}} \rho_{\mathrm{s}} c_{\mathrm{s}} / R$, where $k_{\mathrm{y}}$ is the wave vector in binormal direction, $\rho_{\mathrm{s}}=c_{\mathrm{s}} m_{\mathrm{D}} c /\left(e B_{\text {unit }}\right)$ and $c_{\mathrm{s}}=\sqrt{T_{e} / m_{D}}$ with $m_{D}$ being the mass of deuterium and $B_{\text {unit }}=(1 / r) \partial_{r}\left[1 /(2 \pi) \int \mathbf{B} d \mathbf{S}\right]$. Here, the operator describing the spatial derivative along the field line is formally replaced by a parallel wave number $k_{\|}$and treated as a numerical factor proportional to $1 /(q R)$. Similarly, a Krook collision operator is included, with a generic collision frequency $\nu(E, \lambda)$ with the energy $E$ and the pitch angle parameter $\lambda=v_{\perp}^{2} /\left(v^{2} B(r, \theta) / B_{\text {unit }}(r)\right)$. The generalized potential $\tilde{U}_{k} \equiv \tilde{\phi}_{k}-\left(v_{\|} / c\right) \tilde{A}_{\|, k}$ consists of the fluctuating electrostatic potential $\tilde{\phi}_{k}$ and the parallel component of the fluctuating vector potential $\tilde{A}_{\|, k}$. The effect of the compressional Alfvén dynamics has been computed for typical tokamak parameters used in this work and turns out to impact the results in a negligible way (less than $2 \%$ for the highest $\beta_{\mathrm{e}}$ values considered here). Therefore it is neglected in the present work. However, it is worth noting that parallel magnetic field fluctuations can have an important influence at the very high values of $\beta_{\mathrm{e}}$ obtained in spherical tokamaks, as it has been shown in [29]. We use the simplified $s-\alpha$ geometry model of circular flux surfaces [23]. In this model, the perpendicular $\nabla B$ and curvature drifts are combined in the drift frequency $\omega_{d, k}=\omega_{D, k}\left[\left(v_{\|}^{2}+v_{\perp}^{2} / 2\right) / v_{t h, \sigma}^{2}\right][\cos \theta+(s \theta-\alpha \sin \theta) \sin \theta]$.

In the following, we will focus on the electron dynamics and therefore drop the species notation whenever possible. The radial electron particle flux, comprising both $\mathrm{E} \times \mathrm{B}$ and magnetic flutter, is given by 


$$
\Gamma_{e}=\operatorname{Re} \sum_{k}\left\langle\int d^{3} v J_{0, \sigma} \tilde{h}_{k}^{*}\left(\frac{c}{B} \mathbf{e}_{\|} \times \nabla \tilde{U}_{k}\right) \nabla r\right\rangle_{F S}=\operatorname{Re} \sum_{k}\left\langle\int d^{3} v J_{0, \sigma} \tilde{h}_{k}^{*}\left(i k_{\mathrm{y}} \rho_{\mathrm{s}} c_{\mathrm{s}} \hat{U}_{k}\right)\right\rangle_{F S}
$$

Here, the normalization $\hat{U}_{k}=e \tilde{U}_{k} / T_{\mathrm{e}}$ has been included. The $\langle\ldots\rangle_{F S}$ operator denotes the flux surface average and the asterisk ${ }^{*}$ ' the complex conjugate. Then, Eq. (1) can be formally solved for $\tilde{h}_{k, \sigma}$, and the result can be used in Eq. (2) to find an analytical expression for the particle flux. Direct electromagnetic effects caused by $\left(v_{\|} / c\right) \tilde{A}_{\|}$on trapped electrons can be considered small due to the small average parallel velocity produced by the fast bouncing. Therefore, for trapped electrons, electrostatic expressions, like those derived in [30] remain applicable, and indirect electromagnetic effects on trapped particle fluxes are produced by the change of the real eigenfrequency and the linear growth rate caused by the inclusion of a finite $\beta_{\mathrm{e}}$. These effects are similar to those identified on impurity transport [31]. These effects, however, remain only a minor correction in electron particle transport, as compared with the impact of the additional terms due to $\left(v_{\|} / c\right) \tilde{A}_{\|}$for passing particles. Thus, in our analytical derivations we focus on the passing electron response. A formal solution of Eq. (1) for $\tilde{h}_{k}$ reads

$$
\begin{aligned}
\tilde{h}_{k, \sigma} & =\frac{N_{\mathrm{p}, k, \sigma}}{D_{\mathrm{p}, k, \sigma}} F_{0, \sigma} J_{0, \sigma} \hat{U}_{k}, \\
N_{\mathrm{p}, k, \sigma} & =\left\{\omega_{\mathrm{r}, k}+i \gamma_{k}-\omega_{D, k} / Z_{\sigma}\left[\frac{R}{L_{\mathrm{n}}}+\left(\frac{E}{T}-\frac{3}{2}\right) \frac{R}{L_{\mathrm{T}}}\right]\right\}\left\{\omega_{\mathrm{r}, k}-k_{\|} v_{\|}-\frac{\omega_{d, k}}{Z_{\sigma}}-i\left(\gamma_{k}+\nu_{k}\right)\right\} \\
D_{\mathrm{p}, k, \sigma} & =\left(\gamma_{k}+\nu_{k}\right)^{2}+\left(\omega_{\mathrm{r}, k}-k_{\|} v_{\|}-\omega_{d, k} / Z_{\sigma}\right)^{2} .
\end{aligned}
$$

In order to compare the expression containing $\hat{A}_{\|}$through $\hat{U}$ with the electrostatic one in former works $[7,30,32]$, it is instructive to reformulate the phase correlation between fluctuating density and the generalized potential $\hat{U}$ used for the electromagnetic radial electron flux. Thus, we rewrite Eq. (2) in a form containing only $\tilde{\phi}$ such that the electromagnetic effects caused by $\tilde{A}_{\|}$are explicitly included in the phase shift. This in turn allows us to underline the actual electromagnetic ingredients in the physics. To this purpose, a relation between $\hat{A}_{\|}$and $\hat{\phi}$ is required. This can be obtained using the Fourier transformed Ampère's law for the parallel component of the vector potential, given by

$$
k_{\perp}^{2} \tilde{A}_{\|, k}=-\frac{4 \pi e}{c} \sum_{\sigma} \int d^{3} v v_{\|} Z_{\sigma} \tilde{f}_{\sigma, k} J_{0, \sigma}
$$


where $k_{\perp}$ is the perpendicular wave number. Keeping in mind that only the non-adiabatic part of $\tilde{f}_{\sigma, k}=\tilde{h}_{\sigma, k}-F_{0, \sigma} Z_{\sigma} e \tilde{\phi} / T_{\sigma}$, namely $\tilde{h}_{\sigma, k}$, produces a net current, and taking Eq. (3) for the passing particles, the relation between $\tilde{A}_{\|, k}$ and $\hat{\phi}_{k}$ reads $\hat{A}_{||, k}=\left(c / c_{\mathrm{s}}\right) \hat{\Omega}_{k} \hat{\phi}_{k}=$ $\left(c / c_{\mathrm{s}}\right)\left(\hat{\Omega}_{\mathrm{r}, k}+i \hat{\Omega}_{\mathrm{i}, k}\right) \hat{\phi}_{k}$, with

$$
\hat{\Omega}_{k}=\frac{\beta_{\mathrm{e}} \sum_{\sigma} \int d^{3} v v_{\|} / c_{\mathrm{s}}\left(-Z_{\sigma} N_{\mathrm{p}, k, \sigma} / D_{\mathrm{p}, k, \sigma}\right) F_{0, v} J_{0, \sigma}}{2\left(k_{\perp} \rho_{\mathrm{s}}\right)^{2}+\beta_{\mathrm{e}} \sum_{\sigma} \int d^{3} v v_{\|}^{2} / c_{\mathrm{s}}^{2}\left(-Z_{\sigma} N_{\mathrm{p}, k, \sigma} / D_{\mathrm{p}, k, \sigma}\right) F_{0, v} J_{0, \sigma}},
$$

where $F_{0, v}=F_{0} / n_{0}$. From this description it is apparent that $\beta_{\mathrm{e}}$ is the parameter implying perpendicular magnetic field fluctuations. In present day tokamaks this parameter can reach relatively large values, typically from $0.6 \%$ to $1.3 \%$ in hybrid scenario operation, like in the experiments presented in [33]. Thus, the absolute value of $\hat{\Omega}_{k}$ is if the order of a few percent. At this point it is also important to discuss the symmetry properties of the additional terms originating from fluctuations in $\hat{A}_{\|}$and contributing to Eq. (2) with respect to the ballooning angle $\theta$, because the expression of the particle flux contains the integration over $\theta$ in the flux surface average. Since $\hat{\phi}_{k}$ is symmetric in $\theta$ and $\hat{A}_{\|, k}$ is antisymmetric, $\hat{\Omega}_{k}$ must be antisymmetric as well.

Introducing the trapped particle fraction $f_{t}$, the expression for the radial particle flux caused by passing electrons reads

$$
\begin{aligned}
\Gamma_{p}= & \left(1-f_{t}\right) \sum_{k}\left\langle k_{\mathrm{y}} \rho_{\mathrm{s}} c_{\mathrm{s}} \int d^{3} v\left[1-2 \hat{v}_{\|} \hat{\Omega}_{\mathrm{r}, k}+\hat{v}_{\|}^{2}\left(\hat{\Omega}_{\mathrm{r}, k}^{2}+\hat{\Omega}_{\mathrm{i}, k}^{2}\right)\right] F_{0} J_{0, \mathrm{e}}^{2}\left|\hat{\phi}_{k}\right|^{2} \times\right. \\
& \left.\frac{\left(\hat{\gamma}_{k}+\hat{\nu}_{k}\right) k_{\mathrm{y}} \rho_{\mathrm{s}}\left[R / L_{\mathrm{n}}+\left(E / T_{e}-3 / 2\right) R / L_{\mathrm{Te}}\right]-\left[\hat{\gamma}_{k}\left(\hat{k}_{\| \mid} \hat{v}_{\|}+\hat{\omega}_{d, k}\right)-\hat{\omega}_{r, k} \hat{\nu}_{k}\right]}{\left(\hat{\omega}_{r, k}+\hat{k}_{\| \mid} \hat{v}_{\|}+\hat{\omega}_{d, k}\right)^{2}+\left(\hat{\gamma}_{k}+\hat{\nu}_{k}\right)^{2}}\right\rangle_{F S},
\end{aligned}
$$

where all the frequencies have been normalized to $c_{\mathrm{s}} / R$ and velocities to $c_{\mathrm{s}}$. The sign convention is such that a positive value of $\hat{\omega}_{r}$ denotes a mode propagating in the ion diamagnetic direction, that is an ITG. Compared to former works [7], the new terms in the expression of the flux are those proportional to $\hat{\Omega}_{\mathrm{r}, k}$ and $\hat{\Omega}_{\mathrm{i}, k}$.

The calculation of $\left|\tilde{U}_{k}\right|^{2}$ yields the three terms in the first square bracket at the right hand side of Eq. (6), where the electrostatic component, and the two electromagnetic components, at the first and the second power of $v_{\|}$, and given respectively by $-2 \operatorname{Re}\left(\tilde{\phi}_{k}^{*} \tilde{A}_{\|, k}\right) v_{\|} / c$, and by $\left|\tilde{A}_{\mid, k}\right|^{2}\left(v_{\|} / c\right)^{2}$, can be easily identified. 
It is important to note that the radial particle flux is not linear in the logarithmic gradients of density and temperature due to the implicit dependence of $\hat{\omega}_{r}$ and $\hat{\gamma}$ on the gradients. In addition $\hat{\Omega}$ contains $R / L_{\mathrm{n}}$ and $R / L_{\mathrm{T}}$ as well, providing a new source of nonlinearity. However, Eq. (6) can be still decomposed, and allows the identification of diffusive, thermodiffusive and convective parts due to the explicit occurrence of terms proportional $R / L_{\mathrm{n}}$ and $R / L_{\mathrm{T}}$ or terms not explicitly proportional to any gradient, originating from the right hand side of the gyrokinetic Eq. (1).

In order to check the relative importance of electromagnetic effects on passing and trapped electrons, a standard case of ITG dominated microturbulence for a plasma of deuterons and electrons is defined. That case is used throughout this Section unless specified otherwise. The local parameters are the inverse aspect ratio $r / R=0.17$, the safety factor $q=1.4$ with a magnetic shear of $s=(r / q) \partial_{r} q=0.8$, the normalized logarithmic temperature gradients for deuterons and electrons $R / L_{\mathrm{Ti}}=R / L_{\mathrm{Te}}=9 \equiv R / L_{\mathrm{T}}$ and the deuterium to electron temperature ratio $T_{\mathrm{i}} / T_{\mathrm{e}}=1$.

Fig. (1) shows the fluxes computed in GYRO linear simulations at a single $k_{\mathrm{y}} \rho_{\mathrm{s}}=0.1$ (where the maximum of the linear wave number spectrum of $\gamma /<k_{\perp}^{2}>$ for the cases considered in Section III is located), with different values of $R / L_{\mathrm{n}}$ in circular geometry neglecting the effects of pressure gradients on the magnetic equilibrium $(\alpha=0)$, as a function of the pitch angle parameter $\lambda$. The normalization has been taken in such a way that the total flux is given by the integral over $\lambda$, i.e. $\Gamma=\int_{0}^{\lambda_{\max }} \Gamma(\lambda) d \lambda$. The transition from passing to trapped particles is denoted by the black vertical line. From the $\beta_{\mathrm{e}}=0$ curves it is apparent that the passing particles do not produce a significant flux since they are nearly adiabatic. Thus, the total flux is mainly given by the trapped particles, and it is inward for low $R / L_{\mathrm{n}}$, Fig. 1(a, b), while it points outward for large $R / L_{\mathrm{n}}$, Fig. 1(c). At low logarithmic density gradients, the thermodiffusive and convective trapped electron fluxes are directed inwards and dominate, while at higher $R / L_{\mathrm{n}}$ the increasing contribution of the outward directed diffusive term together with a reduced thermodiffusion (due to the fact that the mode frequency of the most unstable mode moves towards the electron drift 
direction) reverses the direction of the electron flux. This behavior is qualitatively the same also for trapped electrons at finite $\beta_{\mathrm{e}}$, see the total electromagnetic flux in Fig. (1). The latter is calculated as the sum of the $\mathrm{E} \times \mathrm{B}$ and the magnetic flutter contribution $(\mathrm{Fl})$, which is usually very small for the trapped particle fraction. The differences with respect to the trapped electrostatic fluxes are, as mentioned previously, due to an increase of $\hat{\omega}_{\mathrm{r}, k}$ in the ion diamagnetic direction and a decrease of $\hat{\gamma}_{k}$ with increasing $\beta_{\mathrm{e}}$.

The physics behind the strong push of the electron flux in the outward direction by including electromagnetic effects is the loss of adiabaticity of the passing particle fraction as compared to the electrostatic cases. This can be seen in Fig. (1). Electromagnetic passing particle fluxes are directed outwards as much as the trapped fluxes are directed inwards. Thus, the resultant total flux is strongly reduced in the inward direction or even reversed at finite $\beta_{\mathrm{e}}$, as it will be shown in Section III. This behavior can be explained within the analytical description. To this end, Eq. (6) is rewritten in a more explicit and simple form for passing electrons which have $\lambda=0$ already providing the full qualitative information. It reads

$$
\begin{aligned}
\Gamma_{p}= & \left(1-f_{t}\right) \sum_{k}\left\langle k_{\mathrm{y}} \rho_{\mathrm{s}} c_{\mathrm{s}} n_{0} \sum_{\varsigma= \pm 1} \frac{1}{\sqrt{\pi}} \int_{0}^{\infty} d \epsilon \sqrt{\epsilon} \exp (-\epsilon)\left[1-2 \varsigma \sqrt{2 \epsilon \mu} \hat{\Omega}_{\mathrm{r}, k}+4 \epsilon \mu\left(\hat{\Omega}_{\mathrm{r}, k}^{2}+\hat{\Omega}_{\mathrm{i}, k}^{2}\right)\right]\right. \\
& \left.\times \frac{\left(\hat{\gamma}_{k}+\hat{\nu}_{k}\right) k_{\mathrm{y}} \rho_{\mathrm{s}}\left[R / L_{\mathrm{n}}+(\epsilon-3 / 2) R / L_{\mathrm{T}}\right]-\left[\hat{\gamma}_{k}\left(\varsigma \sqrt{2 \epsilon \mu} / q+\hat{\omega}_{d, k}\right)-\hat{\omega}_{r, k} \hat{\nu}_{k}\right]}{\left(\hat{\omega}_{r, k}+\varsigma \sqrt{2 \epsilon \mu} / q+\hat{\omega}_{d, k}\right)^{2}+\left(\hat{\gamma}_{k}+\hat{\nu}_{k}\right)^{2}}\left|\hat{\phi}_{k}\right|^{2}\right\rangle_{F S} .
\end{aligned}
$$

The sign of the velocity in the parallel direction is included using $\varsigma= \pm 1$. We put $\epsilon=$ $E / T_{e}=m_{e} v_{e}^{2} /\left(2 T_{e}\right)$, and we approximated $J_{0}\left(k_{\perp} v_{\perp} / \Omega_{c, e}\right)=1$. Here and in the following derivations, the parallel wave number is put to $k_{\|}=1 /(R q)$. The high value of the deuterium to electron mass ratio is given by $\mu=m_{D} / m_{e}$.

Electromagnetic contributions in Eq. (7) coming from $\tilde{A}_{\|, k}$ can be still identified in the second and third term in the first square bracket at the right hand side. In order to identify the dominant electromagnetic contributions to the particle flux, we observe that the product $\epsilon \mu$, occurring both at the numerator and at the denominator in Eq. (7), is usually much 
larger than unity, unless particles with energies much smaller than the thermal energy are considered. However the relative magnitude of the electromagnetic terms for those particles in Eq. (7) remains small due to the smallness of $\beta_{\mathrm{e}}$, and the contribution to the total particle flux of particles at very low energy has small weight over the energy integral. We turn therefore to consider passing particles whose energy is of the order of the thermal energy, and for which $\epsilon \mu$ is large.

Inserting Eq. (5) in Eq. (7), it is found that most of the contributions to the total flux are very small due to the deuterium to electron mass ratio in the denominator. In particular, it is clear that the electrostatic passing electron flux, which is determined by Eq. (7) imposing $\hat{\Omega}_{\mathrm{r}, k}=\hat{\Omega}_{\mathrm{i}, k}=0$, is close to the null due to the fact that the large slab term in the denominator $(\propto \epsilon \mu)$ is not balanced in the numerator, where the strongest contribution is $\propto \sqrt{\epsilon \mu}$. This provides an almost adiabatic response and agrees with the simulation results of Fig. (1).

In the case of a finite $\beta_{\mathrm{e}}$, the situation changes. The reason for this is that the dominant term in the mass ratio $\mu$ at the denominator can be balanced by finite $\beta_{\mathrm{e}}$ terms in the numerator. In particular, we observe that the term proportional to $\left(\hat{\Omega}_{\mathrm{r}, k}^{2}+\hat{\Omega}_{\mathrm{i}, k}^{2}\right)$ is linear in $\epsilon \mu$ and therefore of the same order as the denominator. However, at the same time this term is of order $\beta_{\mathrm{e}}^{2}$, and does not give the major contribution to the flux, even when multiplied by the $\sqrt{\epsilon \mu}$ term, coming from the $v_{\|} k_{\|}$at the left hand side of the gyrokinetic equation, Eq. (1), in the last square bracket of the numerator in Eq. (7). The dominant electromagnetic contribution to the particle flux comes from the second term $2 \varsigma \sqrt{2 \epsilon} \mu \hat{\Omega}_{\mathrm{r}, k}$ in the first square bracket in the right hand side, when it multiplies the $\hat{k}_{\|} \hat{v}_{\| \mid}$term, that is $\hat{\gamma}_{k} \varsigma \sqrt{2 \epsilon \mu} / q$ in the last square bracket at the right hand side of Eq. (7), resulting in a term of order $\mathcal{O}\left(\beta_{\mathrm{e}} \epsilon \mu\right)$, that is of the same order in $\mu$ as the leading term at the denominator, and yields an outward directed contribution proportional to $\beta_{\mathrm{e}}$. Thus, the dominant electromagnetic contribution to the particle flux is of pure convective type, since not provided by any term directly proportional to a density or temperature gradient, and it is produced by the phase shift between electrostatic potential and electromagnetic potential fluctuations, due to the non- 
adiabatic motion of passing electrons, in combination with the parallel streaming of the passing electrons described by the slab term $\hat{k}_{||} \hat{v}_{\|}$. Moreover it is not caused specifically by the resonance in the denominator of Eq. (7), but it is present over the full velocity space of the passing electrons. A deeper demonstration of this last point is shown in the Appendix, also with the help of linear gyrokinetic calculations with GYRO. Here, on the basis of the derivation performed so far, we consider of interest to discuss the following points.

\section{Separation of particle fluxes into their $\mathbf{E} \times \mathbf{B}$ and magnetic flutter compo- nents}

It is instructive to separate electromagnetic fluxes into their $\mathrm{E} \times \mathrm{B}$ and magnetic flutter (Fl) components in an analytical way. Taking Eq. (2), the phase correlation of the non-adiabatic part of $\tilde{h}_{k}$ with the electrostatic potential $\tilde{\phi}_{k}$ and with the parallel component of the vector-potential $\tilde{A}_{\|, k}$ gives the $\mathrm{E} \times \mathrm{B}$ and flutter contributions, respectively. Therefore, using

$$
\hat{U}_{k}=\left[1-\frac{v_{\|}}{c_{\mathrm{s}}}\left(\hat{\Omega}_{\mathrm{r}, k}+i \hat{\Omega}_{\mathrm{i}, k}\right)\right]\left[\hat{\phi}_{\mathrm{r}, k}+i \hat{\phi}_{\mathrm{i}, k}\right]
$$

and keeping only the leading order terms in the deuterium to electron mass ratio, we rewrite Eq. (7) as follows,

$$
\Gamma_{\mathrm{p}, \mathrm{E} \times \mathrm{B}}=\left(1-f_{t}\right) \sum_{k}\left\langle k_{\mathrm{y}} \rho_{\mathrm{s}} c_{\mathrm{s}} n_{0}\left[2 q\left(k_{\mathrm{y}} \rho_{\mathrm{s}} \hat{\Omega}_{\mathrm{i}, k} R / L_{\mathrm{n}}+\hat{\gamma}_{k} \hat{\Omega}_{\mathrm{r}, k}+\hat{\omega}_{\mathrm{r}, k} \hat{\Omega}_{\mathrm{i}, k}\right)\right]\left|\hat{\phi}_{k}\right|^{2}\right\rangle_{F S}
$$

and

$$
\Gamma_{\mathrm{p}, \mathrm{Fl}}=\left(1-f_{t}\right) \sum_{k}\left\langle k_{\mathrm{y}} \rho_{\mathrm{s}} c_{\mathrm{s}} n_{0}\left[2 q\left(-k_{\mathrm{y}} \rho_{\mathrm{s}} \hat{\Omega}_{\mathrm{i}, k} R / L_{\mathrm{n}}+\hat{\gamma}_{k} \hat{\Omega}_{\mathrm{r}, k}-\hat{\omega}_{\mathrm{r}, k} \hat{\Omega}_{\mathrm{i}, k}\right)\right]\left|\hat{\phi}_{k}\right|^{2}\right\rangle_{F S}
$$

Here, a term proportional to the temperature gradient does not contribute after the integration over energy and is therefore left out in both expressions. From Eq. (9) and Eq. (10) we realize that the total passing flux, i.e. the sum of both contributions, recovers the result of Eq. (7) in the same limit of large $\epsilon \mu$, since the terms proportional to $\hat{\Omega}_{i}$ in the two equations balance exactly. Therefore, the total electromagnetic passing 
particle flux, i.e. the sum of the $\mathrm{E} \times \mathrm{B}$ and the flutter contribution, has no explicit dependence on the logarithmic gradients of density and temperature in leading order of $\mu$, and therefore it is of pure convective type, as already concluded, while both the $\mathrm{E} \times \mathrm{B}$ and flutter contributions separately exhibit a direct dependence on the logarithmic density gradient.

At this point it is instructive to simplify the relation between $\hat{A}_{\|}$and $\hat{\phi}$, Eq. (5). For the $\mathrm{E} \times \mathrm{B}$ component of the flux, only the electron current is considered while the ion current is neglected. This approximation is valid since the electron current gives the dominant contribution to Ampére's law, especially in the cases of large real eigenfrequencies and growthrates (for $\hat{\omega}_{r}, \hat{\gamma}$ of the order of one, the electron current contribution is typically one order of magnitude larger than the ion current contribution). Moreover, only the leading order terms proportional to $\epsilon \mu$ are taken into account. Then the dominant part of the relation between $\hat{A}_{\|, k}$ and $\hat{\phi}_{k}$ can be written as

$$
\hat{\Omega}_{k}=\frac{q \beta_{\mathrm{e}}\left(k_{\mathrm{y}} \rho_{\mathrm{s}} R / L_{\mathrm{n}}+\hat{\omega}_{r}+i \hat{\gamma}\right)}{\left(k_{\perp} \rho_{\mathrm{s}}\right)^{2}} .
$$

Note that again a term proportional to the temperature gradient, namely $k_{\mathrm{y}} \rho_{\mathrm{s}}(\epsilon-3 / 2) R / L_{\mathrm{T}}$, has been left out since it does not give a contribution after integration over energy. The real part of Eq. (11), $\hat{\Omega}_{\mathrm{r}}$, is proportional to $\hat{\omega}_{r}$ and therefore a positive number for ITG modes, while it can become negative for TEM modes. The imaginary part $\hat{\Omega}_{\mathrm{i}}$ is always a positive number independent of the sign of the real frequency.

For the flutter component, only the ion current contribution in Ampére's law has to be considered, since the electron current alone leads to $\Gamma_{\mathrm{p}, \mathrm{Fl}}=0$, as it can be deduced combining Eq. (10) with Eq. (11). In the denominator of Eq. (5), the integral proportional to $\hat{v}_{\|}^{2}$ is small compared to $2\left(k_{\perp} \rho_{\mathrm{S}}\right)^{2}$ and is therefore neglected, such that Eq. (5) can be written as 


$$
\begin{aligned}
\hat{\Omega}_{k} \approx & -\frac{\beta_{\mathrm{e}}}{\left(2 k_{\perp} \rho_{\mathrm{s}}\right)^{2}} \int d^{3} v F_{0, v} \hat{v}_{\|}\left\{\hat{\gamma}(\hat{\gamma}+\hat{\nu})+\left(\hat{\omega}_{r}-\hat{\omega}_{*}\right)\left(\hat{\omega}_{r}-\hat{k}_{\| \mid} \hat{v}_{\|}-\hat{\omega}_{d}\right)\right. \\
& \left.+i\left[\hat{\gamma}\left(\hat{\omega}_{r}-\hat{k}_{\| \mid} \hat{v}_{\|}-\hat{\omega}_{d}\right)-(\hat{\gamma}+\hat{\nu})\left(\hat{\omega}_{r}-\hat{\omega}_{*}\right)\right]\right\}\left\{\left(\hat{\omega}_{r}-\hat{k}_{\| \mid} \hat{v}_{\|}-\hat{\omega}_{d}\right)^{2}+(\hat{\gamma}+\hat{\nu})^{2}\right\}^{-1},
\end{aligned}
$$

where $\hat{\omega}_{*}=k_{\mathrm{y}} \rho_{\mathrm{s}}\left[R / L_{\mathrm{n}}+(\epsilon-3 / 2) R / L_{\mathrm{T}}\right]$. For this expression it can be shown that the real part of $\hat{\Omega}$ is proportional to $-\hat{\omega}_{r}$ while the imaginary part is a positive number (proportional to $1 / \hat{\gamma}$ ).

By comparing the expressions of the ExB, Eq. (9) together with Eq. (11), and the flutter components, Eq. (10) together with Eq. (12), of the passing particle flux, we note that the flutter component is approximately one order of magnitude smaller compared to the $\mathrm{E} \times \mathrm{B}$. As explained above, this is due to the fact that the electron current provides the dominant contribution in Ampére's law. The direction of $\mathrm{E} \times \mathrm{B}$ and flutter fluxes, inward or outward, depends on the interplay among the signs of $\hat{\omega}_{\mathrm{r}}$ and $\hat{\Omega}_{\mathrm{r}}$, which can change depending on the type of instability, and the relative magnitude of the various terms (we remind that $\hat{\Omega}_{\mathrm{i}}$ is always positive). In particular, for ITG modes $\hat{\omega}_{\mathrm{r}}>0, \hat{\Omega}_{\mathrm{r}}>0$ and $\hat{\Omega}_{\mathrm{i}}>0$, we see that the three components of the $\mathrm{E} \times \mathrm{B}$ passing particle flux in Eq. (9) are all directed outward, acting in the same direction. Instead, the three magnetic flutter components are in opposite directions and partly balance each other. The inward directed terms $\propto \hat{\Omega}_{\mathrm{i}}$ are larger than that $\propto \hat{\Omega}_{\mathrm{r}}$, directed outward, and the flutter flux is slightly inward. In contrast, for sufficiently large TEM real frequencies in absolute value, $\hat{\Omega}_{\mathrm{r}}$ becomes negative, and $\mathrm{E} \times \mathrm{B}$ passing electron flux can be directed inward, when the terms $\hat{\gamma} \hat{\Omega}_{\mathrm{r}}+\hat{\omega}_{\mathrm{r}} \hat{\Omega}_{\mathrm{i}}$ exceed $k_{\mathrm{y}} \rho_{\mathrm{s}} \hat{\Omega}_{\mathrm{i}} R / L_{\mathrm{n}}$ in Eq. (9). The flutter component is directed outwards since the term $-k_{\mathrm{y}} \rho_{\mathrm{s}} \hat{\Omega}_{\mathrm{i}} R / L_{\mathrm{n}}$ in the flutter expression Eq. (10) is usually smaller than the other two. For very large density gradients in TEM turbulence, however, the situation is reversed and the $\mathrm{E} \times \mathrm{B}$ passing electron flux is directed outwards while the flutter is directed inwards, in agreement with simulation results. 
In conclusion, for ITG modes the $\mathrm{E} \times \mathrm{B}$ passing flux is always outward and significant, while the magnetic flutter is inward and usually smaller in size. For TEMs with large real frequencies in absolute value, the situation is reversed, and the $\mathrm{E} \times \mathrm{B}$ passing flux can become directed inward, while the magnetic flutter part becomes directed outward. The reversal of the convective part of the $\mathrm{E} \times \mathrm{B}$ flux for modes propagating in the electron drift direction with respect to the ion drift direction has also been found in the fluid description of [11]. However, as we shall show, and differently from ITG modes, the electromagnetic contribution of passing electrons to the particle flux in the case of TEMs is usually small as compared to the one produced by trapped electrons.

\section{Impact of shear and $\alpha$}

The understanding of the role of parallel dynamics in the electromagnetic description of particle fluxes is deepened by studying the separate impact of the parameter s and $\alpha$ in the $s-\alpha$ model. Fig. (2) shows the GYRO computations of real eigenfrequency $\omega_{r}(\mathrm{a})$, linear growthrate $\gamma(\mathrm{b})$, normalized particle $T_{i} \Gamma_{e} / Q_{i}(\mathrm{c})$ and normalized electron to ion heat flux $Q_{e} / Q_{i}(\mathrm{~d})$ as a function of $(s-\alpha)$ for the logarithmic density gradient $R / L_{\mathrm{n}}=$ 3 and a single poloidal wave number $k_{\mathrm{y}} \rho_{\mathrm{s}}=0.1$. Both electrostatic and electromagnetic dependences have been evaluated changing $\alpha$ while keeping the shear constant $(s=0.8$, circles) or vice versa, i.e. scanning shear at $\alpha=0$ (squares), respectively. For $(s-\alpha)$ values larger than $\approx 0.6, \omega_{r}$ as well as $\gamma$ show almost the same values regardless of the parameter which was changed, see Fig. $2(\mathrm{a}, \mathrm{b})$. At $(s-\alpha)<0.6$ and varying shear, stronger deviations to the behavior obtained with changing $\alpha$ can be found. The electrostatic $(s-\alpha)$ dependence of the electron flux, Fig. 2(c), is in both cases (fixed shear or fixed $\alpha$ ) nearly the same, while the electromagnetic dependence shows strong differences. A similar conclusion for the heat flux dependence can be drawn, Fig. 2(d). This can be understood from the analytical derivation. Since the relation between $A_{\|}$ and $\phi$ is given by the Ampère's law, Eq. (4), the perpendicular wave number $k_{\perp}$ plays an essential role to determine the magnitude of $\hat{\Omega}$, see Eq. (5). Within the $s-\alpha$ model, 
shear and pressure gradient enter in different ways in the expression for $k_{\perp}$, since the shear is multiplied by the extended ballooning angle $\theta$ and $\alpha$ by $\sin \theta$ (an expression of $k_{\perp}$ is given in Eq. (13) in Section III). Thus, there is a substantial difference in $k_{\perp}$ by either varying shear or $\alpha$ when the eigenfunctions become extended in $\theta$, that is along the field line, as it is generally the case at finite $\beta_{\mathrm{e}}$, due to the non-adiabatic passing electron dynamics.

\section{Effect of finite collisionality}

From the physical point of view, electromagnetic effects imply radial magnetic field fluctuations which effectively decrease the otherwise fast parallel velocity of passing electrons by rapid radial displacements of their positions. A finite collisionality acts in the same way and therefore also leads to non-adiabaticity. But unlike a finite $\beta_{\mathrm{e}}$, the latter enters through diffusive and thermodiffusive effects, which gives an inward contribution at small $R / L_{\mathrm{n}}$. For the convective part, an additional flux is generated via finite collisionality being directed outwards with ITG as the most unstable mode, as shown in $[7,30,34]$. The same considerations are true for the trapped particle fraction.

\section{Electromagnetic electron fluxes with trapped electron modes}

Lastly, linear gyrokinetic simulations for microturbulence in the TEM domain are performed. To this purpose we chose an electron logarithmic temperature gradient $R / L_{\mathrm{Te}}=9$ while the other gradients are $R / L_{\mathrm{Ti}}=R / L_{\mathrm{n}}=3$ and other parameters remain as in the ITG standard case. Figure (3) shows the electrostatic and electromagnetic components of the passing and trapped particle fluxes. The most important difference compared to the ITG case is the fact that the trapped particle flux in the case of TEM becomes much larger than the passing particle flux, in both electrostatic and electromagnetic cases. Thus, the impact on the particle flux due to a finite value of $\beta_{\mathrm{e}}$ can be expected to remain small in the case of TEMs. Moreover, it is apparent that already at $\beta_{\mathrm{e}}=0$ the passing flux is finite. The reason for this behavior is the 
fact that the mode real eigenfrequency is negative and thus the slab resonance in the denominator of Eq. (7) is obtained at higher particle energy which in turn leads to more particles encountering non-adiabaticity. The electromagnetic $\mathrm{E} \times \mathrm{B}$ component is still slightly positive but rather small due to the fact that $\hat{\Omega}_{\mathrm{r}}$ changes sign which leads to counteracting terms in Eq. (9). However, due to the stronger non-adiabaticity of low energetic passing particles the total $\mathrm{E} \times \mathrm{B}$ flux remains slightly inwards. The total electromagnetic passing flux contribution is almost at the null since the outward flutter component balances the $\mathrm{E} \times \mathrm{B}$ one. Trapped particles are directed more inwards with increasing $\beta_{\mathrm{e}}$ due to changes in the real eigenfrequency and growthrate. These considerations are in agreement with the previous discussions based on the analytical results.

In conclusion, the dominant term providing an outward push of the electron particle flux in ITG turbulence in which electromagnetic effects are included, i.e. Ampère's law due to finite $\beta_{\mathrm{e}}$, has been identified. This is given by the phase shift between electrostatic potential fluctuation and magnetic vector fluctuations produced by the non-adiabatic dynamics of passing electrons, in combination with the compression of their parallel streaming. As such, this dominant transport mechanism is of pure convective type, that is, it is not directly proportional to a logarithmic density or temperature gradient. It is significant over the entire energy range, and is mainly carried by $\mathrm{E} \times \mathrm{B}$ transport, while magnetic flutter transport provides a smaller, inward directed, contribution. For TEM with large real frequencies in absolute value, the situation can be reversed, with the $\mathrm{E} \times \mathrm{B}$ transport being directed inward, while the magnetic flutter part is directed outward. However, electromagnetic passing particle fluxes in case of TEM instabilities remain small as compared to the mainly electrostatic trapped electron fluxes. These can be indirectly affected by changes of the real frequency and the growth rate produced by finite $\beta_{\mathrm{e}}$.

In Appendix A the dependence of the particle flux on energy is illustrated in further detail, also by means of GYRO numerical simulations. 


\section{GYROKINETIC INVESTIGATIONS OF ELECTROMAGNETIC RADIAL ELECTRON PARTICLE FLUXES}

When both particle sources and neoclassical transport are negligible, the condition of turbulent particle flux at the null can be applied to compute the stationary logarithmic density gradient as a function of $\beta$, keeping fixed all the other local plasma parameters.

The stationary normalized logarithmic density gradient $R / L_{\mathrm{n} \text {,stat }}$ is defined as the value of $R / L_{\mathrm{n}}=-(R / n) \partial_{r} n$ where the turbulent particle flux $\Gamma_{e}=0$. As anticipated in Section I, a variation of $\beta$ is considered having two effects. One is the electromagnetic effect connected with the fluctuations of the magnetic field, which are given by the solution of the Ampère's law, and in which $\beta_{\mathrm{e}}$ appears directly as scaling parameter for the strength of the magnetic field fluctuations. The second effect is purely geometrical (electrostatic) and it is related to the role of the radial derivative of the total plasma pressure $\partial_{r} p$ in the magnetic equilibrium and consequently in the expression of the curvature drift. For given logarithmic gradients of the temperature and density profiles, this term is proportional to the total plasma $\beta$.

The quasi-linear calculations in this Section are performed using the same standard ITG case in $s-\alpha$ geometry as before. With the linear version of the gyrokinetic code GYRO particle fluxes as a function of $R / L_{\mathrm{n}}$ are computed. In all the quasi-linear calculations the particle flux $\Gamma$ is normalized to the ion heat flux $Q_{\mathrm{i}}$. Fig. (4) shows the electromagnetic gyrokinetic numerical calculations of the quasi-linear electron particle fluxes as a function of the logarithmic density gradient $R / L_{\mathrm{n}}$ for both $\beta_{\mathrm{e}}=0$, as well as for $\beta_{\mathrm{e}}=0.5 \%$. The resultant fluxes consist of the sum of $\mathrm{E} \times \mathrm{B}$ and magnetic flutter contributions. Two different models for the wave number spectrum of the saturation amplitude of the electrostatic potential are used. Following the works of [32,35], we use either the fully computed poloidal wave number spectrum (12 points from $k_{\mathrm{y}} \rho_{\mathrm{s}}=0.05$ to $k_{\mathrm{y}} \rho_{\mathrm{s}}=1.5$ in logarithmic spacing) with weighting due to $\gamma /\left\langle k_{\perp}^{2}\right\rangle$, see Fig. (4a), or the model proposed in [36] which assumes an exponential decrease of the saturation amplitude of the potential at $k_{\mathrm{y}} \rho_{\mathrm{s}}$ smaller or larger than the $k_{\mathrm{y}}$ value at which $\gamma /\left\langle k_{\perp}^{2}\right\rangle$ is at the maximum, see Fig. (4b). This exponential 
shape of the saturation amplitude was based on measurements of the density fluctuations at high wave numbers [37]. In the $s-\alpha$ model, the perpendicular wave number is defined as $k_{\perp}=\sqrt{k_{x}^{2}+k_{\mathrm{y}}^{2}\left[1+(s \theta-\alpha \sin \theta)^{2}\right]}$ such that $\left\langle k_{\perp}^{2}\right\rangle$ is given by

$$
\left\langle k_{\perp}^{2}\right\rangle=k_{x}^{2}+k_{\mathrm{y}}^{2}\left[1+\left\langle(s \theta-\alpha \sin \theta)^{2}\right\rangle\right]
$$

where

$$
\left\langle(s \theta-\alpha \sin \theta)^{2}\right\rangle=\frac{\int(s \theta-\alpha \sin \theta)^{2}\left|\tilde{\phi}_{k}(\theta)\right|^{2} d \theta}{\int\left|\tilde{\phi}_{k}(\theta)\right|^{2} d \theta}
$$

with $\theta$ being the extended ballooning angle and $\tilde{\phi}$ the fluctuating electrostatic potential. We find that $\gamma /\left\langle k_{\perp}^{2}\right\rangle$ has its maximum around $k_{\mathrm{y}} \rho_{\mathrm{s}} \approx 0.1$ (slightly shifted towards higher values for low $R / L_{\mathrm{n}}$ and towards lower values at high $R / L_{\mathrm{n}}$ and $\beta_{\mathrm{e}}$ ), and strongly decreases with increasing poloidal wave number such that the ratio of the maximum value of $\gamma /\left\langle k_{\perp}^{2}\right\rangle$ to the value at $k_{\mathrm{y}} \rho_{\mathrm{s}}=0.5$ is of order 100 , as also found in [32].

The gyrokinetic calculations in Fig. (4) show that with a finite $\beta_{\mathrm{e}}$ the fluxes are pushed in the outward direction for $R / L_{\mathrm{n}}<3$ while they are only weakly affected at high $R / L_{\mathrm{n}}$, as already pointed out in Section II. Such a behavior leads to a reduction of the logarithmic density gradient at which the flux is zero. This effect is larger in the case where $\alpha=0$ (open circles) compared to the case in which it is included consistently with the value of $\beta_{\mathrm{e}}=0.5 \%$ (open squares). A finite experimentally relevant collisionality, here $\left(R / c_{\mathrm{s}}\right) \nu_{\mathrm{ei}}=0.06$, has also the effect of pushing the fluxes in the outward direction, see diamond symbols in Fig. (4). This is consistent with the discussion in Section II. From the collisional curve with $\beta_{\mathrm{e}}=0.5 \%$ we realize that the wave number model proportional to $\gamma /\left\langle k_{\perp}^{2}\right\rangle$ predicts a value of $R / L_{\mathrm{n}}$ close to the null.

Using these $R / L_{\mathrm{n}}$ scans, the value of the logarithmic density gradient at the null of the fluxes can be identified, and computed for increasing values of $\beta$. The influence of a finite $\beta_{\mathrm{e}}$ on the logarithmic density gradient at zero electron particle flux is presented in Fig. (5) for the two different models for the saturation amplitude of the wave number spectrum. The cases with concentric circles (a), with a self-consistent value of $\alpha(\mathrm{b})$ and with finite 
collisionality of $\left(R / c_{\mathrm{s}}\right) \nu_{\mathrm{ei}}=0.06$ using concentric circles for the magnetic equilibrium (c) are considered. We see that in the first case the decrease of the stationary logarithmic density gradient with increasing $\beta_{\mathrm{e}}$ is weak at low values of $\beta_{\mathrm{e}}$ while it becomes strong for $\beta_{\mathrm{e}}$ approaching $0.5 \%$. The inclusion of a consistent value of $\alpha$ reduces this effect. Also in the collisional case, the drop of $R / L_{\mathrm{n}, \mathrm{stat}}$ is more linear. It is interesting to note that the model proposed in [36] predicts generally larger $R / L_{\mathrm{n}, \text { stat }}$ than the $\gamma /\left\langle k_{\perp}^{2}\right\rangle$ model at high values of $\beta_{\mathrm{e}}$, while at low $\beta_{\mathrm{e}}$ both models show similar values in the collisionless cases. The reason for this behavior is the fact that fluxes at high $k_{\mathrm{y}} \rho_{\mathrm{s}}$, which are directed inwards have a larger weight in the model of [36]. Moreover, for increasing $\beta_{\mathrm{e}}$ the high $k_{\mathrm{y}}$ contributions become larger in the inward direction while they are quite small at $\beta_{\mathrm{e}}=0$. It is also of interest to note that, using a corresponding circular Miller geometry [38] at the place of the $s-\alpha$ model geometry, the predicted stationary density gradient decreases by about $10 \%$. These results motivate further investigations of the poloidal spectrum of particle fluxes and a detailed comparison of linear with nonlinear gyrokinetic calculations in realistic geometry. We address this point in the next Section for parameters close to those measured in ASDEX Upgrade.

\section{COMPARISON OF QUASI-LINEAR AND NONLINEAR ELECTROMAGNETIC ELECTRON PARTICLE FLUXES}

In Section III it has been pointed out that the logarithmic density gradient $R / L_{\mathrm{n}}$, at which the particle flux is at the null, decreases with increasing $\beta_{\mathrm{e}}$ in Ampère's equation. However, it was shown that different choices of the wave number spectra of the fluxes provide different $R / L_{\mathrm{n}, \text { stat }}$. The main goal of this Section is the calculation of finite $\beta$ effects on the peaking of the density profile containing both electromagnetic and geometrical implications on a realistic case. In addition, we compute the stationary logarithmic density gradients using nonlinear simulations. The comparison with linear calculations helps to identify a quasi-linear model which predicts $R / L_{\mathrm{n}, \text { stat }}$ in good agreement with nonlinear results. 
To this purpose, calculations of electron transport including electromagnetic effects are performed for $\mathrm{H}$-mode parameters around mid-radius using the Miller geometry model for the magnetic equilibrium. The choice of dimensionless parameters which are applied in the gyrokinetic simulations are typical of an ASDEX Upgrade plasma and therefore should be considered more realistic than the reference cases discussed previously. The equilibrium parameters are given by $r / a=0.56, R / a=3.53, q=1.33, s=1.12$, Shafranov shift $\Delta=$ -0.11 , elongation $\kappa=1.38$ with elongation shear $s_{\kappa}=(r / \kappa) \partial_{r} \kappa=0.11$, and triangularity $\delta=0.032$ with triangularity shear $s_{\delta}=r \partial_{r} \delta=0.055$. The local parameters for the two species plasma consisting of deuterons and electrons are $R / L_{\mathrm{Ti}}=6.13, R / L_{\mathrm{Te}}=6.13$, $T_{i} / T_{e}=1, \beta_{\mathrm{e}}=0.65 \%$. The collisionality is $\left(R / c_{\mathrm{s}}\right) \nu_{\mathrm{ei}}=0.067$, and $Z_{\mathrm{eff}}=2$. The value of $\beta_{\mathrm{e}}$ is scanned while the magnetic equilibrium was held constant.

In linear simulations, the dominant micro-instability in these conditions is an ITG mode for $k_{\mathrm{y}} \rho_{\mathrm{s}} \lesssim 0.7$. Beyond that value a TEM mode is found. We choose a spectrum from $k_{\mathrm{y}} \rho_{\mathrm{s}}=0.05-1.5$ with 12 points logarithmically spaced. A sufficiently high number of radial simulation points is taken in order to keep the radial resolution $d x / \rho_{\mathrm{s}} \lesssim 0.5$. For nonlinear flux tube simulations, we took a spectrum from $k_{\mathrm{y}} \rho_{\mathrm{s}}=0.04-2.68$ using 64 toroidal modes. The simulation box is chosen to be $L_{x} / \rho_{\mathrm{s}}=82$ and $L_{y} / \rho_{\mathrm{s}}=148$. Using 216 radial points we obtain a radial resolution of $d x / \rho_{\mathrm{s}}=0.38$. Moreover, we use a grid of 256 points in the velocity space ( 8 energies, 8 passing and 8 trapped pitch angles and two signs of velocity).

Figure (6) shows the nonlinear spectrum of electrostatic and electromagnetic particle fluxes at $R / L_{\mathrm{n}}=2$. It is found that while the position of the peak for both the electrostatic flux and the electromagnetic $\mathrm{E} \times \mathrm{B}$ flux component remains at $k_{\mathrm{y}} \rho_{\mathrm{s}} \approx 0.2$, the former is directed less strong in the outward direction compared to the latter. The peak of the electromagnetic flutter component is at slightly higher $k_{\mathrm{y}} \rho_{\mathrm{s}}$ and the flux is negative over the full range of the poloidal spectrum.

Figure (7a) shows the results for the stationary logarithmic density gradient using linear and nonlinear simulations at increasing $\beta_{\mathrm{e}}$ in Ampère's equation. Electromagnetic fluxes consist of the sum of $\mathrm{E} \times \mathrm{B}$ and magnetic flutter components. For the quasi-linear fluxes, 
different wave number spectra have been used for comparative reasons, i.e. fluxes according to the maximum of $\gamma /\left\langle k_{\perp}^{2}\right\rangle$ and to the maximum of $\gamma$ (in the ITG branch), wave number spectra according to $\left(\gamma /\left\langle k_{\perp}^{2}\right\rangle\right)^{2}$ and $\gamma /\left\langle k_{\perp}^{2}\right\rangle$ as well as the wave number spectrum proposed in $[36]$.

In linear simulations, the logarithmic density gradient decreases with increasing $\beta_{\mathrm{e}}$ regardless of the wave number spectrum, which is consistent with the results from the parameter study in Section II. However, there are big differences in the predicted values at which the electron flux vanishes. Using the wave number spectral weight due to the maximum in $\gamma /\left\langle k_{\perp}^{2}\right\rangle$, which peaks at $k_{\mathrm{y}} \rho_{\mathrm{s}} \approx 0.18$, or due to the spectral average using $\left(\gamma /\left\langle k_{\perp}^{2}\right\rangle\right)^{2}$ results in a very low positive (or even negative) density gradient, while the wave number due to the maximum in $\gamma$ or the spectrum due to $\gamma /\left\langle k_{\perp}^{2}\right\rangle$ allows for moderate values of $R / L_{\mathrm{n}}$ at vanishing electron flux. Larger stationary gradients are obtained using the wave number spectrum proposed in [36].

Taking the nonlinear simulation results we see that the decrease of $R / L_{\mathrm{n}}$ is quite substantial and rather linear with increasing $\beta_{\mathrm{e}}$. The comparison to quasi-linear results reveals that the wave number spectrum proposed in [36] and $\left(\gamma /\left\langle k_{\perp}^{2}\right\rangle\right)^{z}$ with $z=0.7$ works best for these cases, while the other ones do not deliver satisfying agreements. At $\beta_{\mathrm{e}}$ approaching $1 \%$ the former turn out to give higher stationaty density gradients compared to the nonlinear results. However, it was impossible to obtain meaningful fluxes for $\beta_{\mathrm{e}}>1 \%$ in linear simulations since the reduction of turbulence and subsequently of growthrates due to electromagnetic stabilization led to strong spectral variations of the fluxes such that a adequate comparison of quasi-linear and nonlinear stationary density gradients cannot be made. In order to check the impact of a realistic description of the geometry, the value of $R / L_{\mathrm{n}, \text { stat }}$ at $\beta_{\mathrm{e}}=0.65 \%$ has been computed with a corresponding $s-\alpha$ geometry. It is found that in $s-\alpha$ the value of $R / L_{\mathrm{n}, \text { stat }}$ is reduced by $30 \%$.

A more appropriate and realistic description of the experimental conditions considers not only the effect of $\beta_{\mathrm{e}}$ in the Ampére's equation, but also the impact of $\beta^{\prime}=8 \pi \partial_{r} p / B_{\text {unit }}$ in the curvature drift. To this purpose, Fig. (7b) compares quasi-linear with nonlinear 
simulation results. The experimental stationary logarithmic density gradient at $\beta_{\mathrm{e}}=0.65 \%$ remains unchanged, but in the cases with quasi-linear calculations including averages over the wave number spectrum (open triangles and stars), the consistent inclusion of finite $\beta$ in both the Ampère's law and the magnetic equilibrium shows a much weaker dependence of $R / L_{\mathrm{n} \text {,stat }}$ on $\beta_{\mathrm{e}}$ compared to the case in which only Ampère's equation is affected. This is consistent with the results in Section III, Fig. (5). In nonlinear simulations, we find that the reduction of $R / L_{\mathrm{n} \text {,stat }}$ with increasing $\beta_{\mathrm{e}}$, while changing consistently also $\beta^{\prime}$ in the magnetic equilibrium parameters in input (open circles), is much closer to the result obtained with increasing $\beta_{\mathrm{e}}$ only and keeping $\beta^{\prime}$ fixed (full circles), with respect to the result from quasi-linear simulations. Moreover, it is apparent that the same quasi-linear rules for the wave number spectrum, which reproduced the nonlinear results when only $\beta_{\mathrm{e}}$ is changed in a fairly good way, do not agree with the nonlinear results. The reason for these differences has been investigated and is shown in Fig. 8, for simulations with $R / L_{n e}=2$. In Fig. 8(a), a comparison of $\left|\tilde{\phi}_{k}\right|^{2}$ between the linear (black, full line for $\left(\gamma /\left\langle k_{\perp}^{2}\right\rangle\right)^{0.7}$ and dashed line for spectrum proposed in [36]) and nonlinear wave number spectra (color, circle for $\beta_{\mathrm{e}}=\beta^{\prime}=0$, square for $\beta_{\mathrm{e}}=0$ with $\beta^{\prime}$ consistent to a value if $\beta_{\mathrm{e}}$ was $0.65 \%$, and diamond for $\beta_{\mathrm{e}}=0.65 \%$ with a $\beta^{\prime}$ consistent with $\left.\beta_{\mathrm{e}}=0.65 \%\right)$ are shown for $R / L_{\mathrm{n}}=2$. The normalization is done in such a way that the integral over all binormal wave numbers $k_{y}$ gives one. The main difference between the linear choices and the nonlinear simulation results is the fact that in linear calculations large $k_{y}$ values play an important role while in nonlinear simulations the $k_{y} \rho_{s}$ values around 0.2-0.3 have a much stronger impact. Moreover it is interesting to note that for this case the linear choices for $\left|\tilde{\phi}_{k}\right|^{2}$ do not change in the three cases (maximum of $\gamma /\left\langle k_{\perp}^{2}\right\rangle$ remains at $k_{y} \rho_{s}=0.18$ ) while for the nonlinear cases shifts of the maximum are obtained, namely large scales reveal non-negligible differences in the three cases while the small scale behavior $\left(k_{y} \rho_{s}>0.4\right)$ can be described by the power law $\left|\tilde{\phi}_{k}\right|^{2} \propto k_{y}^{-5}\left(\left|\tilde{\phi}_{k_{y}<1}\right|^{2} \propto k_{y}^{-4.8}\right.$ and $\left.\left|\tilde{\phi}_{k_{y}>1}\right|^{2} \propto k_{y}^{-5.2}\right)$, which is slightly steeper than the $k_{y}^{-4.3}$ result for density fluctuations found in [39].

Such large differences between the spectra of $\left|\tilde{\phi}_{k}\right|^{2}$ applied in the quasi-linear calculations, 
and the actual ones obtained in the nonlinear simulations might lead to the conclusion that the total quasi-linear particle fluxes are always significantly far from the nonlinear ones. However this is not always the case. The reason for this is shown in Fig. 8(b), where the quasi-linear weights $\Im\left(\tilde{n}_{k} \tilde{\phi}_{k}^{*}\right) /\left|\tilde{\phi}_{k}\right|^{2}$ are directly compared with those extracted from the actual nonlinear saturated state. Full symbols show the linear results, whereas open symbols show the results from nonlinear simulations. In the comparison, we observe that a clear difference appears in the high $k_{y} \rho_{s}$ part of the spectrum, where linear results are more strongly directed outwards, and cross the zero line at larger values of $k_{y} \rho_{s}$, as compared with the nonlinear spectra. Moreover, from the comparison between $\beta_{\mathrm{e}}=\beta^{\prime}=0$ (circles) with $\beta_{\mathrm{e}}=0, \beta^{\prime}\left(\beta_{\mathrm{e}}=0.65 \%\right)$ (squares) and $\beta_{\mathrm{e}}=0.65 \%, \beta^{\prime}\left(\beta_{\mathrm{e}}=0.65 \%\right)$ (diamonds) of the linear simulations, the cause of the larger discrepancy between nonlinear and quasi-linear stationary logarithmic density gradients in the case a consistent variation of $\beta^{\prime}$ is included in the simulations, shown in Fig. 7(b), can be identified. At $k_{y} \rho_{s}>0.7$, the $\beta_{\mathrm{e}}=\beta^{\prime}=0$ values are shifted in the outward direction compared to the cases with fixed $\beta^{\prime}$, thus lowering the stationary density gradient, particularly in the case of a wave number spectrum giving a large impact to small scales, like the one proposed in [36].

From the detailed comparisons presented in Fig. (8) it can be concluded that, particularly when the particle flux is close to the null, the inward contribution from the relatively small scales is usually weaker in linear calculations compared to nonlinear simulations, a feature which was already observed in Ref. [34]. Thereby, in these cases, the use of the actual nonlinear spectrum for $\left|\tilde{\phi}_{k}\right|^{2}$ in quasi-linear models does not necessarily provide the most accurate quasi-linear evaluation of the total nonlinear particle flux. A compensation should take place for a more accurate matching of the total flux, where quasi-linear weights, which are too small in size at small scales, have their contribution enhanced in a quasi-linear model in which the corresponding values of the $\left|\tilde{\phi}_{k}\right|^{2}$ spectrum at those scales are relatively larger with respect to the actual nonlinear ones. These considerations can be of particular importance for quasi-linear models of particle transport, since in this transport channel quasi-linear weights usually change their sign from outward to inward with increasing wave 
number, as a consequence of the different impact of collisionality at the different scales [34]. Finally, it has to be noted that in the present comparison, linear results consider only one mode at each wave number, that is the most unstable one, whereas a more appropriate approach, like for instance that undertaken in [36], should take into account the entire spectrum of unstable (or perhaps even stable) modes at each wave number. This indeed can play a role in the establishment of the quasi-linear weights in the nonlinear simulations. In addition, we believe that a strong component in the differences between linear and nonlinear results comes from the impact of wave number (toroidal mode) coupling, which takes place in the nonlinear simulations, but which is not included in the quasi-linear models.

The decrease with increasing $\beta$ of the predicted value of $R / L_{\mathrm{n}, \text { stat }}$ obtained by the nonlinear simulations with consistent variation of $\beta^{\prime}$, presented in Fig. 7(b), remains rather strong, and does not appear to be qualitatively consistent with the weak effect documented so far in non-dedicated experimental studies [8-10]. In order to address this problem within a more realistic approach, we have also considered the impact of a particle source like the one provided by neutral beam injection (NBI) fueling on the theoretically predicted dependence of $R / L_{\mathrm{n}, \mathrm{stat}}$ on $\beta$. This can be done by looking for the value of $R / L_{\mathrm{n}}$ at which a given predicted ratio $\Gamma_{e} T / Q_{\text {tot }}$ matches the corresponding ratio of the volume integral of the particle source to the volume integral of the heat source, instead of more simply the condition $\Gamma_{e}=0$, which assumes no particle source. A rough estimate of the ratio of the volume integral of the particle source density delivered by the beams, $\Gamma_{N B I}$, to the volume integral of the NBI heat power density $Q_{N B I}$ is given by $\Gamma_{N B I} / Q_{N B I}=1 / E_{N B I}$, where $E_{N B I}$ is an effective (averaged) beam ion injection energy. Therefore, in the presence of beam fueling, and in stationary conditions, with negligible neoclassical transport, the value of $R / L_{\mathrm{n} \text {,stat }}$ can be expected to be given by the following condition $\Gamma_{e} T / Q_{t o t} \simeq \Gamma_{N B I} T / Q_{N B I}\left(Q_{N B I} / Q_{t o t}\right) \simeq\left(T / E_{N B I}\right)\left(Q_{N B I} / Q_{t o t}\right)$. At constant plasma density, $\beta \propto T$ and by this we observe that with increasing $\beta$, the value of $\Gamma_{e} T / Q_{t o t}$ at which $R / L_{\mathrm{n}, \text { stat }}$ can be identified, increases as well. Considering that most of the present experiments are heated with intense neutral beam injection, we find of interest to check whether, on the basis of our nonlinear simulations of a realistic ASDEX Upgrade 
case, this source effect is significant in determining an "apparent" $\beta$ dependence (or $\beta$ independence) of the density peaking. For this reason we have taken typical parameters of the ASDEX Upgrade neutral beam injection system, and considered an effective beam energy $E_{N B I}=70 \mathrm{keV}$, which yields, with a toroidal magnetic field of $2.4 \mathrm{~T}$ and a fixed density of $6 \times 10^{-19} \mathrm{~m}^{-3}$, the following scaling $T / E_{N B I}=3.41 \beta_{\mathrm{e}}$. Figure (9) shows the results of this exercise, in which we considered three different fractions of $Q_{N B I} / Q_{t o t}$, that is $0,50 \%$ and $100 \%$. On the basis of the nonlinear simulations presented in this paper, we find that the effect is significant and suggests that plasma discharges in which a large fraction of the heating power is not delivered by NBI systems should observe a stronger decrease of the density peaking with increasing $\beta$ with respect to experiments in which the plasma is heated almost exclusively by beams. In the latter case, a $\beta$ independence or even a moderate increase of the peaking with increasing $\beta$ is predicted.

\section{CONCLUSIONS}

Finite $\beta$ effects on electron transport in tokamak plasmas due to both Ampère's law and changes in the magnetic equilibrium have been investigated using linear and nonlinear gyrokinetic flux tube simulations. The logarithmic density gradient at the null of the turbulent particle flux $R / L_{\mathrm{n} \text {,stat }}$ has been calculated as a function of $\beta_{\mathrm{e}}$. In ITG turbulence, the electromagnetic effect due to the inclusion of fluctuations of the magnetic field in the perpendicular direction implies a non-adiabatic response of the passing electrons, as it was pointed out also in [22]. This produces an outward directed convection and strongly reduces the value of $R / L_{\mathrm{n}, \text { stat }}$ with increasing $\beta_{\mathrm{e}}$. In TEM turbulence the effect is found to be weaker. The physics of the electromagnetic effects on the particle flux has been investigated by means of an analytical derivation, starting from a formal solution of the gyrokinetic equation, and the analytical results have been shown to be fully consistent with the numerical simulations. The contribution due to magnetic flutter transport has been investigated, and has been found to be directed inward in ITG turbulence and non-negligible at the highest values of $\beta_{\mathrm{e}}$. In this 
context, the present gyrokinetic study agrees and extends a previous analysis based on a fluid model [11], and confirms the reversal of the particle flux from inward to outward with increasing $\beta_{\mathrm{e}}$ previously found in nonlinear gyrokinetic simulations of ITG turbulence [12].

Within a more realistic description of the impact of $\beta$ on the density peaking, simulation results have been presented in which also the effect of the total plasma pressure gradient in the curvature drift, due to the modification of the magnetic equilibrium, is included consistently. The two effects are of course strongly combined in experiments, while they can be separated in the theoretical calculations. The present study shows that once both effects are included consistently in a realistic case, the predicted decrease of density peaking with increasing $\beta$ becomes weaker. A significant difference has been found in these cases between quasi-linear results and nonlinear simulations, since in the latter the inclusion of a consistently varying plasma pressure gradient has a more limited impact. In fact, in quasi-linear calculations, the predicted value of the logarithmic density gradient at the null of the particle flux $R / L_{\mathrm{n}, \text { stat }}$ is found to be strongly affected by the assumptions made on the binormal wave number spectrum of the fluctuating potential. It has been shown that contributions to the particle flux at different wave numbers can be directed inward or outward, and therefore require an appropriate weighting rule in quasi-linear models in order to reproduce the total nonlinear flux. A detailed comparison between the quasi-linear and the nonlinear spectra shows that linear calculation based on the most unstable linear mode at each wave number produce contributions to the particle flux at small scales which are significantly more outward or less inward directed with respect to the corresponding nonlinear results. In this context, the predicted value of $R / L_{\mathrm{n}, \text { stat }}$ is an appropriate and experimentally relevant parameter over which the adequacy of the assumptions on the wave number spectrum made in the quasi-linear models can be tested against the nonlinear results.

In order to address at a qualitative level the comparison with the experimental observations, the effect of core fueling by neutral beams has been included in the estimate of the predicted stationary value of the logarithmic density gradient $R / L_{\mathrm{n}, \text { stat }}$. In conditions 
of neutral beam injection heating only, at constant plasma density, the impact of the beam fueling on $R / L_{\mathrm{n}, \mathrm{stat}}$ increases with increasing $\beta$. On the basis of the nonlinear gyrokinetic simulations presented here, for typical ASDEX Upgrade parameters, this is found to have non-negligible consequences on the dependence of the predicted $R / L_{\mathrm{n}, \text { stat }}$ on $\beta$. In condi-

tions of dominant neutral beam injection heating and constant density, $R / L_{\mathrm{n}, \text { stat }}$ is found to become almost independent of $\beta$, in contrast with the prediction of a rather strong decrease of $R / L_{\mathrm{n}, \text { stat }}$ with increasing $\beta$ when core fueling by neutral beams is absent. These theoretical results should motivate further dedicated experimental research on this important aspect for the performance of high $\beta$ tokamak scenarios.

The present results are also relevant in other fields of plasma physics. Due to the importance of the parallel velocity for the behavior of electromagnetic particle fluxes it is emphasized that an electromagnetic kinetic treatment and in particular the consideration of magnetic flutter transport is crucial for small scale turbulence in slab-like astrophysical plasmas, since in particular the value of $\beta$ in interstellar $(\beta \sim 1$, see e. g. [40]) or intergalactic media $(\beta \sim 10-100$, see e. g. [41]) as well as in jet-formation regions at the black hole magnetosphere $(\beta \sim 1-100$, see e. g. [42]), is significantly higher than in tokamak plasmas.

\section{ACKNOWLEDGMENTS}

The authors are grateful to Alberto Bottino and Bruce Scott for fruitful discussions. Parts of the simulation work have been performed on the EFDA super-computer 'High Performance Computing for Fusion (HPC-FF)' in Jülich, Germany.

\section{APPENDIX A: LOSS OF ADIABATICITY FOR ELECTROMAGNETIC PASSING ELECTRONS IN THE FULL ENERGY RANGE}

In order to show the loss of adiabaticity of passing electrons in response to electromagnetic fluctuations over the full energy range, the energy dependences of quasi-linear electrostatic $\left(\beta_{\mathrm{e}}=0\right)$ and electromagnetic $\left(\beta_{\mathrm{e}}=0.5 \%\right)$ electron fluxes are shown in Fig. 
(10). Three different values of $\lambda$ in the passing range have been chosen, and an ITG case with $R / L_{\mathrm{n}}=R / L_{\mathrm{Te}}=0.1$ and all other parameters equal to the standard ITG case introduced in Section II is considered. This choice of parameters allows us to focus on the convective part of the electron particle flux. The normalization is taken in such a way that the resulting quasi-linear fluxes are plotted divided by the function of energy $\sqrt{\epsilon} \exp (-\epsilon)$, which includes the dependence on energy of both the Maxwellian and the Jacobian. This procedure allows the identification of the physics differences between an electrostatic and an electromagnetic description of electron fluxes by focusing on the resonant term in Eq. (6). Figure (10a) shows the quasi-linear electrostatic fluxes for $\lambda=0.02, \lambda=0.44$ and $\lambda=0.79$. Particles at very low energy experience the resonance, see Eq. (6), and therefore give a contribution to the particle flux. However, the comparison with the quasi-linear electromagnetic $\mathrm{E} \times \mathrm{B}$ flux, Fig. (10b), reveals that the electrostatic flux is very low since the electrons are almost adiabatic. The electromagnetic $\mathrm{E} \times \mathrm{B}$ and flutter contributions do not have a strong resonance behavior at low energy, as shown in Fig. (10b, c). Instead they exhibit finite contributions in the full energy range. These numerical results are consistent with the analytical derivations obtained in Section II.

[1] A. C. C. Sips, R. Arslanbekov, C. Atanasiu, W. Becker, G. Becker, K. Behler, K. Behringer, A. Bergmann, R. Bilato, D. Bolshukhin, K. Borrass, B. Braams, M. Brambilla, F. Braun, A. Buhler, G. Conway, D. Coster, R. Drube, R. Dux, S. Egorov, T. Eich, K. Engelhardt, H.-U. Fahrbach, U. Fantz, H. Faugel, M. Foley, K. B. Fournier, P. Franzen, J. C. Fuchs, J. Gafert, G. Gantenbein, O. Gehre, A. Geier, J. Gernhardt, O. Gruber, A. Gude, S. Günter, G. Haas, D. Hartmann, B. Heger, B. Heinemann, A. Herrmann, J. Hobirk, F. Hofmeister, H. Hohenöcker, L. Horton, V. Igochine, D. Jacobi, M. Jakobi, F. Jenko, A. Kallenbach, O. Kardaun, M. Kaufmann, A. Keller, A. Kendl, J.-W. Kim, K. Kirov, R. Kochergov, H. Kollotzek, W. Kraus, K. Krieger, B. Kurzan, P. T. Lang, P. Lauber, M. Laux, F. Leuterer, A. Lohs, 
A. Lorenz, C. Maggi, H. Maier, K. Mank, M.-E. Manso, M. Maraschek, K. F. Mast, P. McCarthy, D. Meisel, H. Meister, F. Meo, R. Merkel, D. Merkl, V. Mertens, F. Monaco, A. Mück, H. W. Müller, M. Münich, H. Murmann, Y.-S. Na, G. Neu, R. Neu, J. Neuhauser, J.-M. Noterdaeme, I. Nunes, G. Pautasso, A. G. Peeters, G. Pereverzev, S. Pinches, E. Poli, M. Proschek, R. Pugno, E. Quigley, G. Raupp, T. Ribeiro, R. Riedl, S. Riondato, V. Rohde, J. Roth, F. Ryter, S. Saarelma, W. Sandmann, S. Schade, H.-B. Schilling, W. Schneider, G. Schramm, S. Schweizer, B. Scott, U. Seidel, F. Serra, S. Sesnic, C. Sihler, A. Silva, E. Speth, A. Stäbler, K.-H. Steuer, J. Stober, B. Streibl, E. Strumberger, W. Suttrop, A. Tabasso, A. Tanga, G. Tardini, C. Tichmann, W. Treutterer, M. Troppmann, P. Varela, O. Vollmer, D. Wagner, U. Wenzel, F. Wesner, R. Wolf, E. Wolfrum, E. Würsching, Q. Yu, D. Zasche, T. Zehetbauer, H.-P. Zehrfeld, and H. Zohm, Plasma Physics and Controlled Fusion, 44, B69 (2002).

[2] T. Luce, M. Wade, J. Ferron, A. Hyatt, A. Kellman, J. Kinsey, R. L. Haye, C. Lasnier, M. Murakami, P. Politzer, and J. Scoville, Nucl. Fusion, 43, 321 (2003).

[3] E. Joffrin, A. Sips, J. Artaud, A. Becoulet, L. Bertalot, R. Budny, P. Buratti, P. Belo, C. Challis, F. Crisanti, M. de Baar, P. de Vries, C. Gormezano, C. Giroud, O. Gruber, G. Huysmans, F. Imbeaux, A. Isayama, X. Litaudon, P. Lomas, D. McDonald, Y. Na, S. Pinches, A. Staebler, T. Tala, A. Tuccillo, K.-D. Zastrow, and J.-E. C. to the Work Programme, Nuclear Fusion, 45, $626(2005)$.

[4] ITER Technical Basis, ITER EDA Documentation Series No. 24 (IAEA, Vienna), Chap. 7 (2002).

[5] D. J. Ward, I. Cook, Y. Lechon, and R. Saez, Fusion Eng. Design, 75-79, 1221 (2005).

[6] M. Greenwald, J. Terry, S. Wolfe, S. Ejima, M. Bell, S. Kaye, and G. Neilsen, Nucl. Fusion, 28, 2199 (1988).

[7] C. Angioni, E. Fable, M. Greenwald, M. Maslov, A. G. Peeters, H. Takenaga, and H. Weisen, Plasma Phys. Controlled Fusion, 51, 124017 (2009). 
[8] H. Weisen, A. Zabolotsky, M. Maslov, M. Beurskens, C. Giroud, D. Mazon, and J.-E. contributors, Plasma Phys. Controlled Fusion, 48, A457 (2006).

[9] C. Angioni, H. Weisen, O. Kardaun, M. Maslov, A. Zabolotsky, C. Fuchs, L. Garzotti, C. Giroud, B. Kurzan, P. Mantica, A. Peeters, J. Stober, and the ASDEX Upgrade Team and contributors to the EFDA-JET Workprogramme, Nucl. Fusion, 47, 1326 (2007).

[10] M. Maslov, C. Angioni, H. Weisen, and JET-EFDA contributors, Nucl. Fusion, 49, 075037 (2009).

[11] A. Eriksson and J. Weiland, Phys. Plasmas, 12, 092509 (2005).

[12] J. Candy, Phys. Plasmas, 12, 072307 (2005).

[13] J. Candy and R. E. Waltz, J. Comput. Phys., 186, 545 (2003).

[14] J. Candy and R. E. Waltz, Phys. Rev. Lett., 91, 045001 (2003).

[15] J. D. Callen, Phys. Rev. Lett., 39, 1540 (1977).

[16] A. B. Rechester and M. N. Rosenbluth, Phys. Rev. Lett., 40, 38 (1978).

[17] B. Scott, Plasma Phys. Controlled Fusion, 39, 1635 (1997).

[18] T. Dannert and F. Jenko, Phys. Plasmas, 12, 072309 (2005).

[19] A. Casati, C. Bourdelle, X. Garbet, F. Imbeaux, J. Candy, F. Clairet, G. Dif-Pradalier, G. Falchetto, T. Gerbaud, V. Grandgirard, Ö. Gürcan, P. Hennequin, J. Kinsey, M. Ottaviani, R. Sabot, Y. Sarazin, L. Vermare, and R. Waltz, Nucl. Fusion, 49, 085012 (2009).

[20] R. E. Waltz, A. Casati, and G. M. Staebler, Phys. Plasmas, 16, 072303 (2009).

[21] C. Angioni, A. G. Peeters, G. V. Pereverzev, F. Ryter, and G. Tardini, Phys. Rev. Lett., 90, 205003 (2003).

[22] V. Naulin, A. Kendl, O. E. Garcia, A. H. Nielsen, and J. J. Rasmussen, Phys. Plasmas, 12, $052515(2005)$. 
[23] J. W. Connor, R. J. Hastie, and J. B. Taylor, Phys. Rev. Lett., 40, 396 (1978).

[24] J. Candy, Plasma Phys. Controlled Fusion, 51, 105009 (2009).

[25] T. M. Antonsen and B. Lane, Physics of Fluids, 23, 1205 (1980).

[26] E. A. Frieman and L. Chen, Physics of Fluids, 25, 502 (1982).

[27] G. Rewoldt, W. M. Tang, and M. S. Chance, Physics of Fluids, 25, 480 (1982).

[28] G. G. Howes, S. C. Cowley, W. Dorland, G. W. Hammett, E. Quataert, and A. A. Schekochihin, The Astrophysical Journal, 651, 590 (2006).

[29] C. Bourdelle, W. Dorland, X. Garbet, G. W. Hammett, M. Kotschenreuther, G. Rewoldt, and E. J. Synakowski, Phys. Plasmas, 10, 2881 (2003).

[30] E. Fable, C. Angioni, and O. Sauter, Plasma Phys. Controlled Fusion, 52, 015007 (2010).

[31] T. Hein and C. Angioni, Phys. Plasmas, 17, 012307 (2010).

[32] E. Fable, C. Angioni, and O. Sauter, Plasma Phys. Controlled Fusion, 50, 115005 (2008).

[33] C. Maggi, R. Groebner, C. Angioni, T. Hein, L. Horton, C. Konz, A. Leonard, C. Petty, A. Sips, P. Snyder, J. Candy, R. Waltz, A. Upgrade, and D.-D. Teams, Nucl. Fusion, 50, $025023(2010)$.

[34] C. Angioni, J. Candy, E. Fable, M. Maslov, A. G. Peeters, R. E. Waltz, and H. Weisen, Phys. Plasmas, 16, 060702 (2009).

[35] F. Jenko, T. Dannert, and C. Angioni, Plasma Phys. Controlled Fusion, 47, B195 (2005).

[36] C. Bourdelle, X. Garbet, F. Imbeaux, A. Casati, N. Dubuit, R. Guirlet, and T. Parisot, Phys. Plasmas, 14, 112501 (2007).

[37] P. Hennequin, R. Sabot, C. Honor, G. T. Hoang, X. Garbet, A. Truc, C. Fenzi, and A. Qumneur, Plasma Phys. Controlled Fusion, 46, B121 (2004). 
[38] R. L. Miller, M. S. Chu, J. M. Greene, Y. R. Lin-Liu, and R. E. Waltz, Phys. Plasmas, 5, 973 (1998).

[39] A. Casati, T. Gerbaud, P. Hennequin, C. Bourdelle, J. Candy, F. Clairet, X. Garbet, V. Grandgirard, O. D. Gürcan, S. Heuraux, G. T. Hoang, C. Honoré, F. Imbeaux, R. Sabot, Y. Sarazin, L. Vermare, and R. E. Waltz, Phys. Rev. Lett., 102, 165005 (2009).

[40] A. Bhattacharjee, C. S. Ng, and S. R. Spangler, The Astrophysical Journal, 494, 409 (1998).

[41] P. P. Kronberg, Phys. Plasmas, 10, 1985 (2003).

[42] S. Koide, D. L. Meier, K. Shibata, and T. Kudoh, The Astrophysical Journal, 536, 668 (2000). 

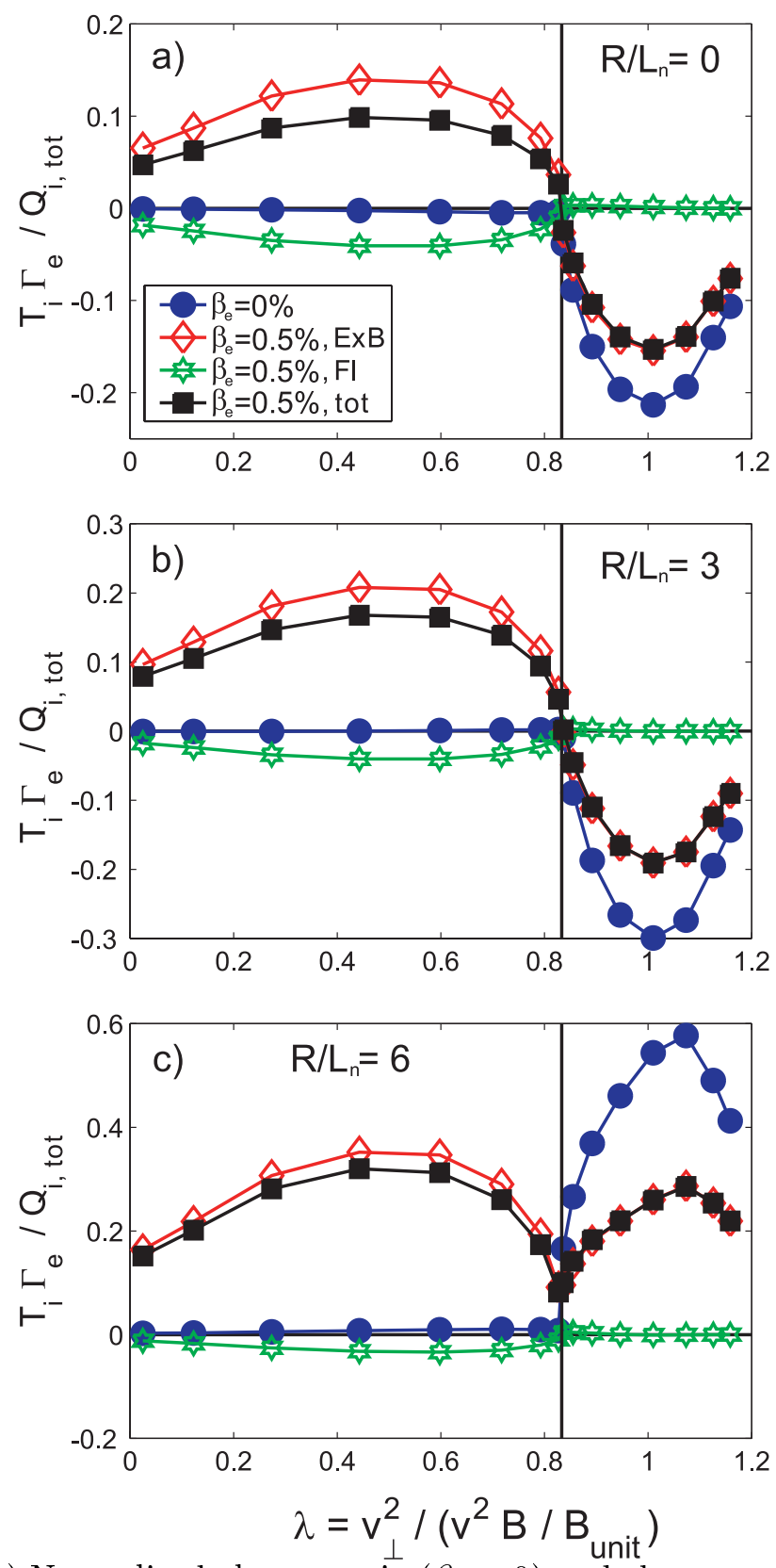

FIG. 1. (Color online) Normalized electrostatic $\left(\beta_{\mathrm{e}}=0\right)$ and electromagnetic $\left(\beta_{\mathrm{e}}=0.5 \%\right)$ linear electron flux as a function of the pitch angle parameter $\lambda$ for the logarithmic density gradient $R / L_{\mathrm{n}}=0$ (a), $R / L_{\mathrm{n}}=3$ (b) and $R / L_{\mathrm{n}}=6$ (c). A single poloidal wavenumber $k_{\mathrm{y}} \rho_{\mathrm{s}}=0.1$ in circular geometry has been chosen. The electron fluxes are normalized to the full velocity space integrated ion heat flux $Q_{\mathrm{i}, \text { tot }}$. Symbols over the curves identify the grid points in $\lambda$ used in the GYRO calculations. 

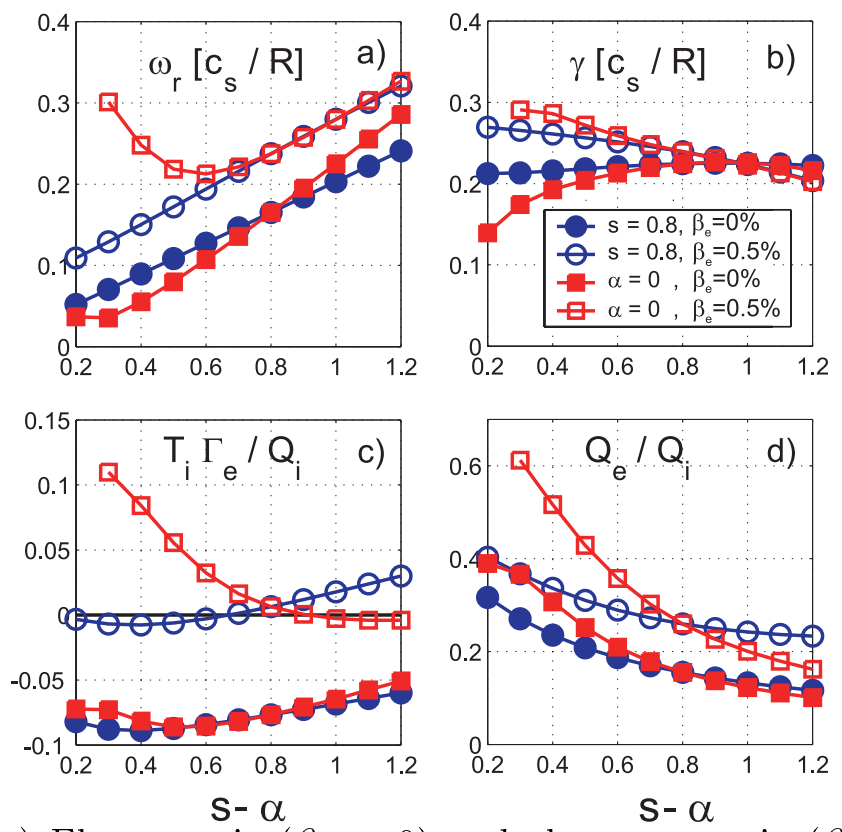

FIG. 2. (Color online) Electrostatic $\left(\beta_{\mathrm{e}}=0\right)$ and electromagnetic $\left(\beta_{\mathrm{e}}=0.5 \%\right)$ dependence of real eigenfrequency $\omega_{r}(\mathrm{a})$, linear growthrate $\gamma(\mathrm{b})$, normalized particle flux $T_{i} \Gamma_{e} / Q_{i}(\mathrm{c})$ and normalized electron to ion heat flux $Q_{e} / Q_{i}(\mathrm{~d})$ as a function of $s-\alpha$ for the logarithmic density $R / L_{\mathrm{n}}=3$ and a single poloidal wave number $k_{\mathrm{y}} \rho_{\mathrm{s}}=0.1$ in circular geometry. 


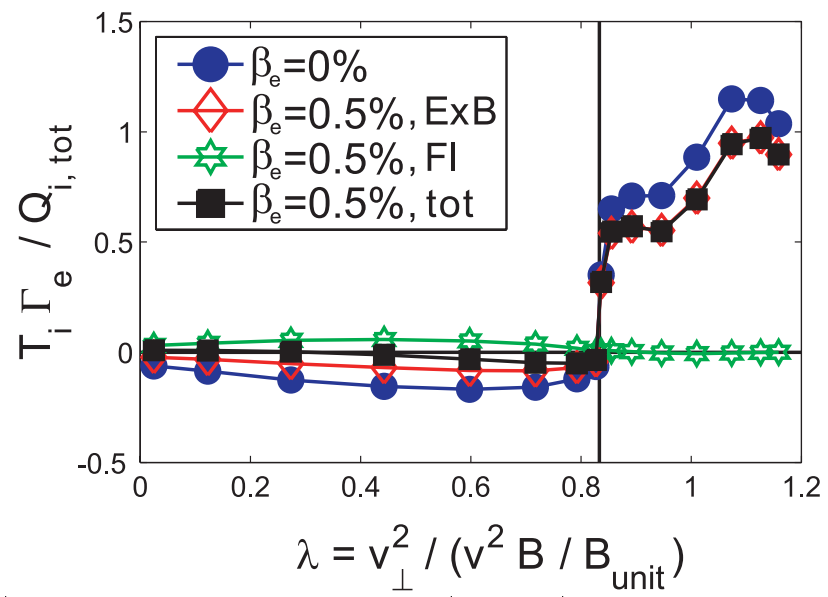

FIG. 3. (Color online) Normalized electrostatic $\left(\beta_{\mathrm{e}}=0\right)$ and electromagnetic $\left(\beta_{\mathrm{e}}=0.5 \%\right)$ linear electron flux as a function of the pitch angle parameter $\lambda$ for a trapped electron mode (TEM) case (see text). A single poloidal wavenumber $k_{\mathrm{y}} \rho_{\mathrm{s}}=0.1$ in circular geometry has been chosen. The electron fluxes are normalized to the full velocity space integrated ion heat flux $Q_{\mathrm{i}, \text { tot }}$. Symbols over the curves identify the grid points in $\lambda$ used in the GYRO calculations. 
Figures
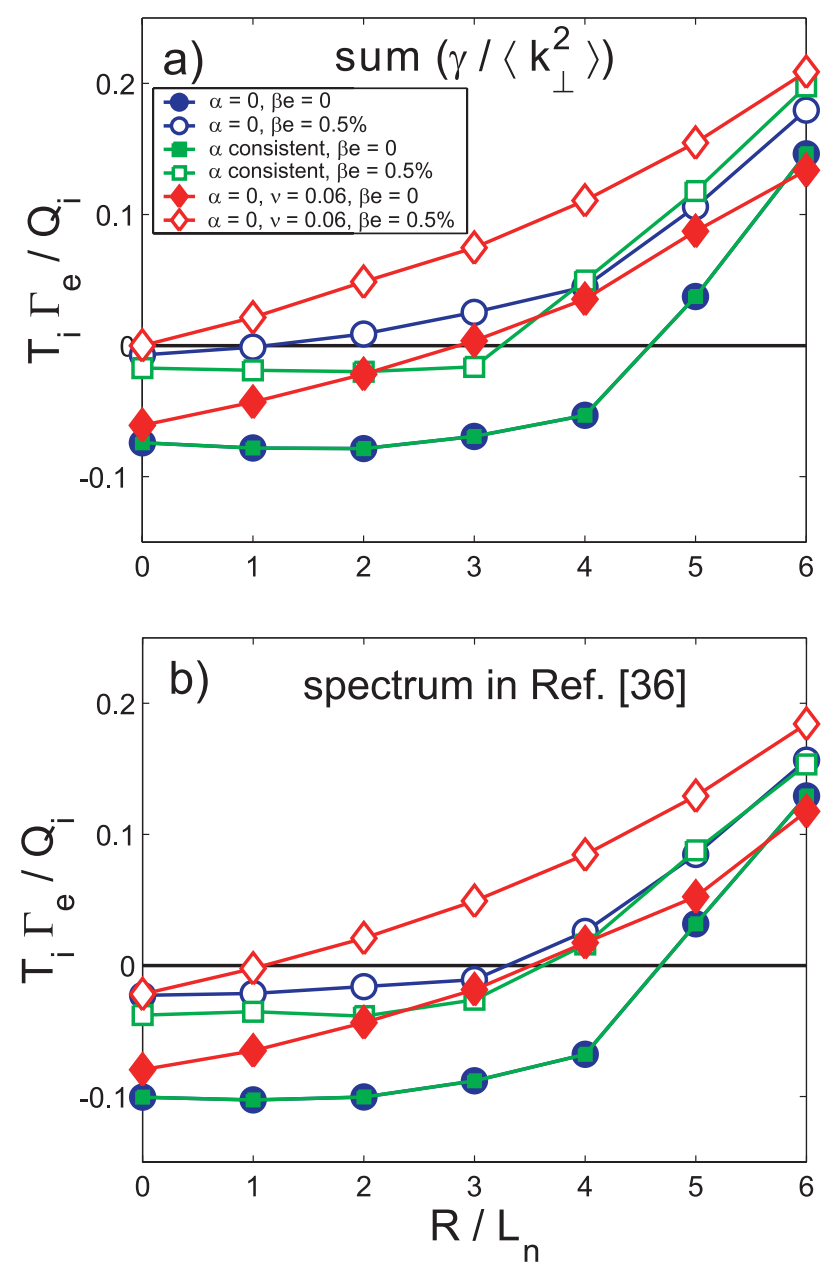

FIG. 4. (Color online) Normalized electrostatic $\left(\beta_{\mathrm{e}}=0\right.$, full symbols) and electromagnetic $\left(\beta_{\mathrm{e}}=0.5 \%\right.$, open symbols) quasi-linear electron particle flux $T_{\mathrm{i}} \Gamma_{\mathrm{e}} / Q_{\mathrm{i}}$ as a function of the logarithmic density gradient $R / L_{\mathrm{n}}$ with $\alpha=0$ and collisionless (circles), with $\alpha$ varied consistently with $\beta$ and collisionless (squares), and with $\alpha=0$ and collisional (diamonds). Wave number spectra proportional to $\gamma /\left\langle k_{\perp}^{2}\right\rangle_{F S}$ (a) or as proposed in [36] (b) have been assumed for the fluxes. 
Figures
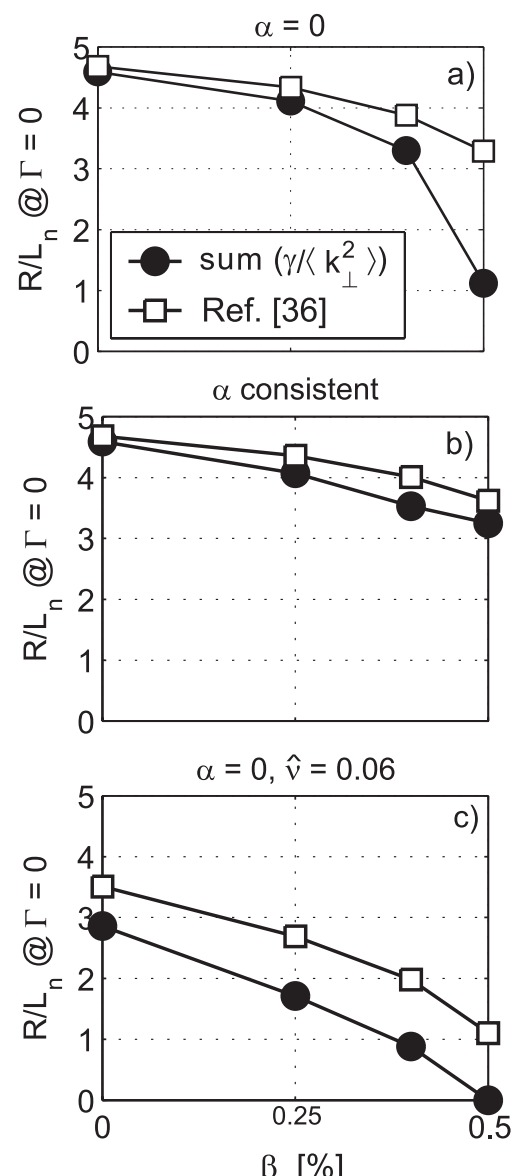

FIG. 5. Electromagnetic dependence of logarithmic density gradient $R / L_{\mathrm{n}}$ at the null of the quasi-linear electron flux as a function of $\beta_{\mathrm{e}}$. A circular case (a), a case with values of $\alpha$ consistent with $\beta$ (b), and a circular case with finite collisionality of $\left(R / c_{\mathrm{s}}\right) \nu_{\mathrm{ei}}=0.06$ (c) with two models for the wave number spectrum of the linear fluxes are considered. 


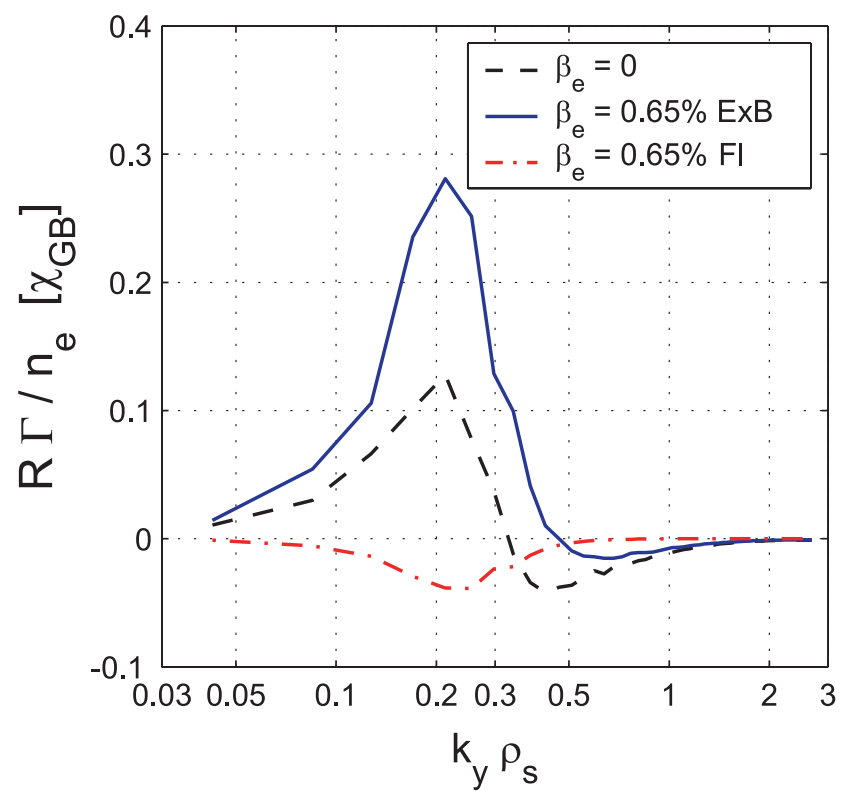

FIG. 6. (Color online) Nonlinear gyro-Bohm normalized electron particle flux spectra for plasma parameters similar to ASDEX Upgrade hybrid discharges (see text). The electrostatic as well as the electromagnetic $\mathrm{E} \times \mathrm{B}$ and magnetic flutter contributions are shown. 

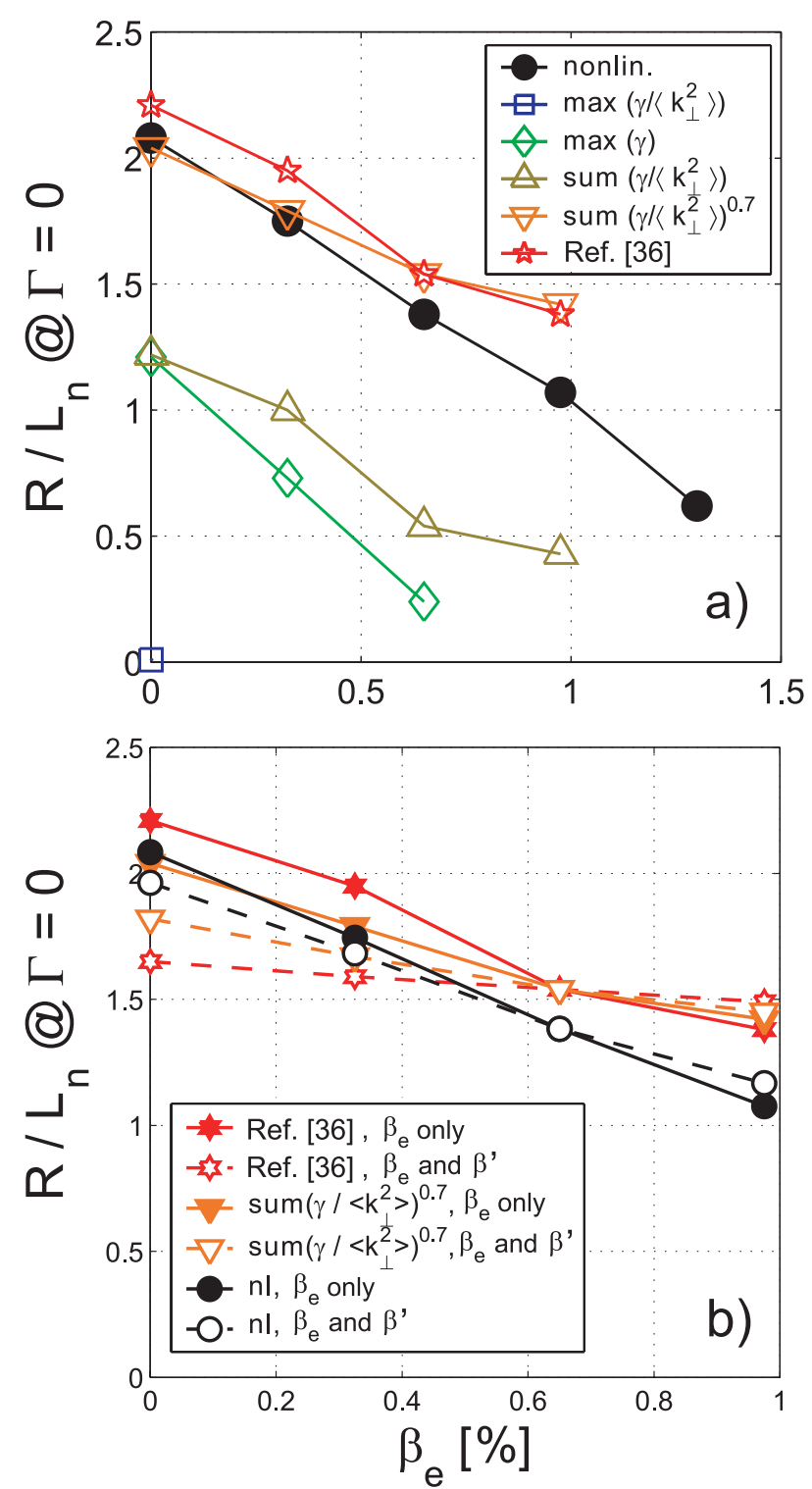

FIG. 7. (Color online) Stationary logarithmic density gradient using nonlinear simulations and quasi-linear calculations with different assumptions for the poloidal wave number spectrum of electron particle fluxes as a function of $\beta_{\mathrm{e}}$ (a). The influences of finite $\beta$ exclusively in Ampère's equation (' $\beta_{\mathrm{e}}$ only') as well as in both Ampère's equation and the consistent magnetic equilibrium $\left(\beta_{\mathrm{e}}\right.$ and $\left.\beta^{\prime}\right)$ on the electron particle fluxes are shown in (b) using quasi-linear and nonlinear calculations. The chosen plasma parameters are similar to ASDEX Upgrade hybrid discharges. 

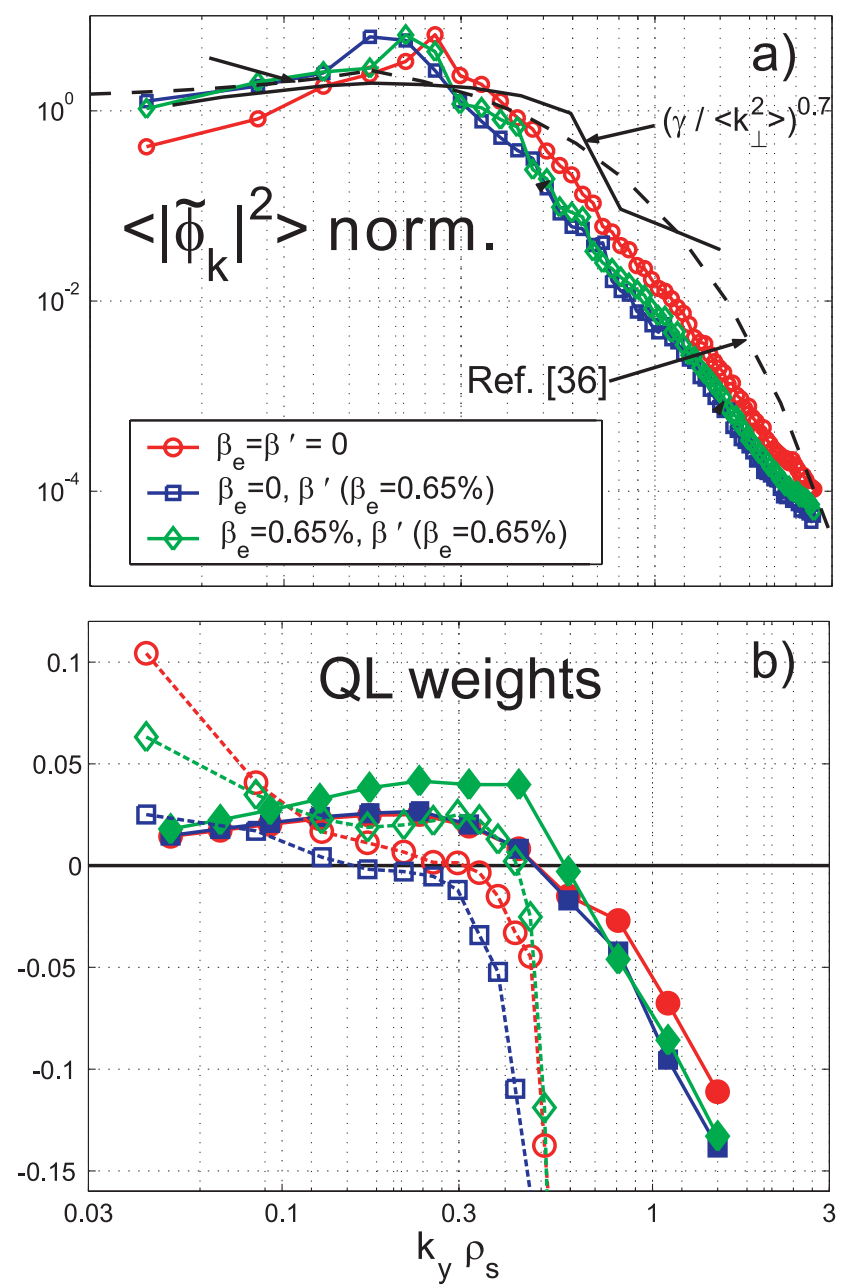

FIG. 8. (Color online) Potential fluctuation spectra $\left|\tilde{\phi}_{k}\right|^{2}$ from nonlinear gyrokinetic simulations (a). Circles denote the case of $\beta_{\mathrm{e}}=\beta^{\prime}=0$, squares stand for $\beta_{\mathrm{e}}=0$ with $\beta^{\prime}$ consistent to a value if $\beta_{\mathrm{e}}$ was $0.65 \%$, and diamonds for $\beta_{\mathrm{e}}=0.65 \%$ with a $\beta^{\prime}$ consistent with a $\beta_{\mathrm{e}}$ of $0.65 \%$. Also the quasi-linear rules are shown. The normalization is $\int d k_{y}\left|\tilde{\phi}_{k_{y}}\right|^{2}=1$. Quasi-linear weights $\Im\left(\tilde{n}_{k} \tilde{\phi}_{k}^{*}\right) /\left|\tilde{\phi}_{k}\right|^{2}$ obtained from linear (continuous lines) and nonlinear (dashed lines) simulations (b). The chosen plasma parameters are similar to ASDEX Upgrade hybrid discharges with $R / L_{\mathrm{n}}=2$. 


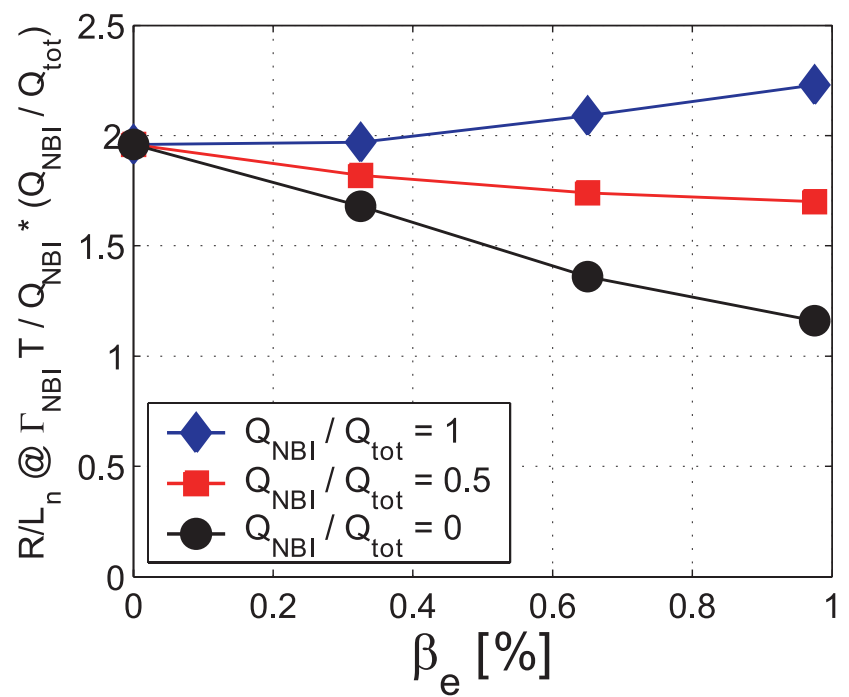

FIG. 9. (Color online) Stationary logarithmic density gradient from nonlinear simulations changing both $\beta_{\mathrm{e}}$ and $\beta^{\prime}$ consistently for three different assumptions of the fraction of neutral beam injection heating to the total heating mix for plasma parameters similar to ASDEX Upgrade hybrid discharges. 

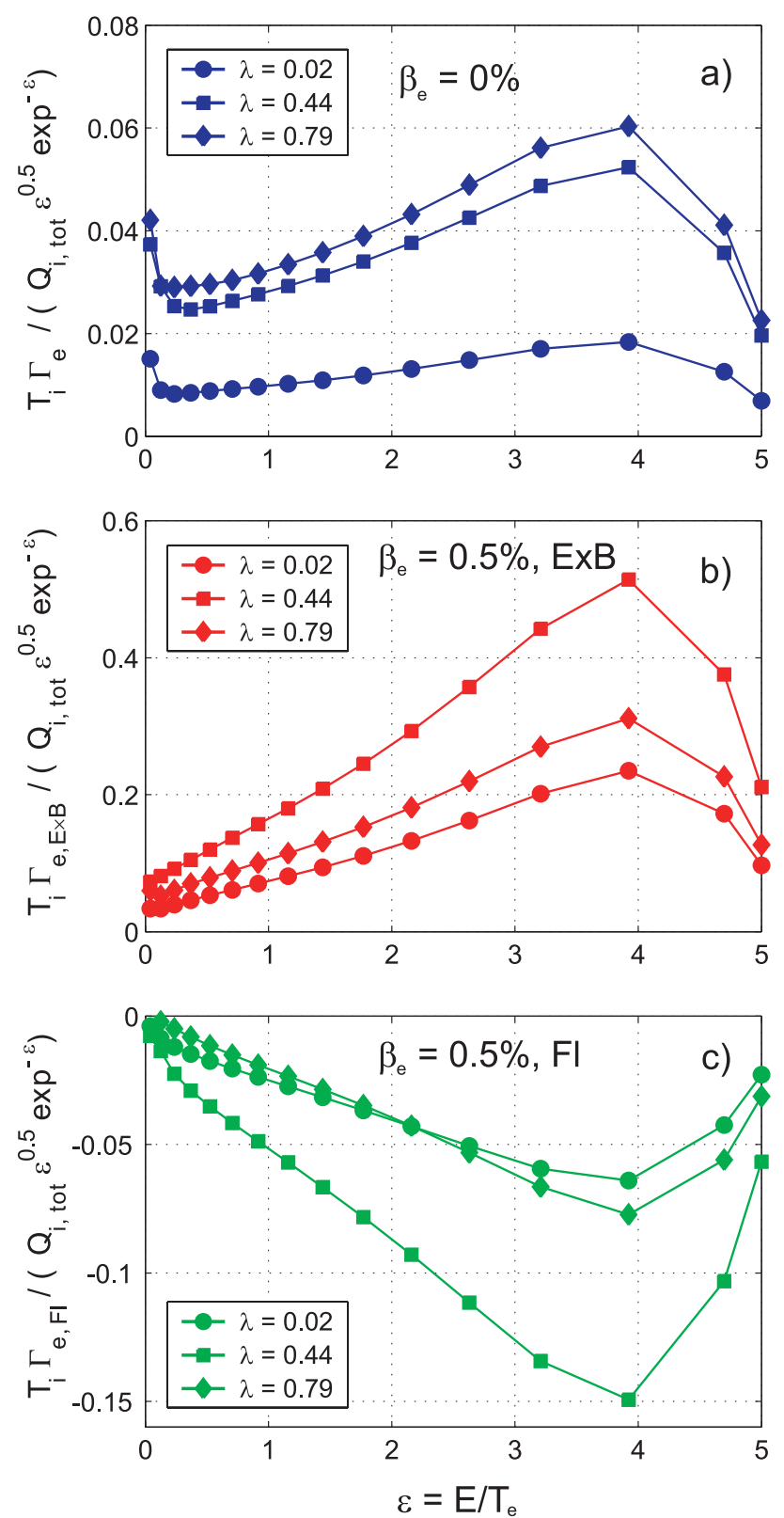

FIG. 10. (Color online) Normalized electrostatic $\left(\beta_{\mathrm{e}}=0, \mathrm{a}\right)$ and electromagnetic $\left(\beta_{\mathrm{e}}=0.5 \%, \mathrm{~b}\right.$, c) quasi-linear electron fluxes as a function of the normalized energy $\epsilon=E / T_{\mathrm{e}}$ for an ITG case with all parameters like the standard ITG case of Section II, except $R / L_{\mathrm{n}}=R / L_{\mathrm{Te}}=0.1$. A single poloidal wavenumber $k_{\mathrm{y}} \rho_{\mathrm{s}}=0.1$ in circular geometry has been chosen. The electron fluxes are normalized to the full velocity space integrated ion heat flux $Q_{\mathrm{i}, \text { tot }}$, and divided by the Jacobian and the Maxwellian. Symbols over the curves identify the grid points in $\epsilon$ used in the GYRO calculations. 